\title{
DIVISIBILITY OF REDUCTION IN GROUPS OF RATIONAL NUMBERS
}

\author{
FRANCESCO PAPPALARDI
}

\begin{abstract}
Given a multiplicative group of nonzero rational numbers and a positive integer $m$, we consider the problem of determining the density of the set of primes $p$ for which the order of the reduction modulo $p$ of the group is divisible by $m$. In the case when the group is finitely generated the density is explicitly computed. Some examples of groups with infinite rank are considered.
\end{abstract}

\section{INTRODUCTION}

It is a well known result due to Hasse [5] and others that the probability that 2 generates a subgroup of $\mathbb{F}_{p}^{*}$ with even order is $17 / 24$ while the probability that 3 generates a subgroup of $\mathbb{F}_{p}^{*}$ with even order is $2 / 3$. So, it might not be a surprise to read that the probability that 2 and 3 together generate a subgroup of $\mathbb{F}_{p}^{*}$ with even order is $195 / 224$ and that the probability that 3 and 5 together generate a subgroup of $\mathbb{F}_{p}^{*}$ with even order is $6 / 7$. In general, groups of rational numbers containing 2 have a slightly higher tendency, than those not containing 2 , to generate subgroups of $\mathbb{F}_{p}^{*}$ with even order. This phenomenon is related to the fact that the size of the Galois group of $x^{8}-2$ is half of the size of the Galois group of $x^{8}-\ell$ where $\ell$ is an odd prime. This paper deals with these properties in a fairly general context.

Let $\Gamma \subset \mathbb{Q}^{*}$ be a multiplicative subgroup and define the support Supp $\Gamma$ of $\Gamma$ to be the set of primes $p$ such that the $p$-adic valuation of some elements of $\Gamma$ is nonzero. In the special case of finitely generated $\Gamma$ (see [15]) it is easy to see that Supp $\Gamma$ is finite. For any prime $p \notin \operatorname{Supp} \Gamma$, we denote by $\Gamma_{p}$ the reduction of $\Gamma$ modulo $p$. That is,

$$
\Gamma_{p}=\{g(\bmod p): g \in \Gamma\} .
$$

It is clear that since $p \notin \operatorname{Supp} \Gamma, \Gamma_{p} \subseteq \mathbb{F}_{p}^{*}$ is a subgroup. As usual we also denote by $\operatorname{ind}_{p}(\Gamma)$ and $\operatorname{ord}_{p}(\Gamma)$ the index and the order of $\Gamma_{p}$. That is,

$$
\operatorname{ord}_{p}(\Gamma)=\# \Gamma_{p} \quad \text { and } \quad \operatorname{ind}_{p}(\Gamma)=\left[\mathbb{F}_{p}^{*}: \Gamma_{p}\right]=(p-1) / \operatorname{ord}_{p}(\Gamma) .
$$

Here, for $m \in \mathbb{Z}$, we consider the function

$$
A_{\Gamma}(x, m)=\#\left\{p \leq x: p \notin \operatorname{Supp} \Gamma, m \mid \operatorname{ord}_{p}(\Gamma)\right\} .
$$

The special case of $\Gamma$ generated by a rational number in $\mathbb{Q}^{*} \backslash\{1,-1\}$ has been extensively considered in the literature. For a complete and updated account we refer to Moree's survey paper [11, Sections 9.2 and 9.3]. Moree [12, Wiertelak [20] and the author [16], give several asymptotic formulas for $A_{\langle g\rangle}(x, m)$ with

Received by the editor October 30, 2012 and, in revised form, May 25, 2013.

2010 Mathematics Subject Classification. Primary 11N37; Secondary 11N56.

This project was supported in part by G.N.S.A.G.A of I.N.D.A.M.. 
$g \in \mathbb{Q}^{*} \backslash\{1,-1\}$. More general results have been considered by Moree [13] and by Chinen and Murata [2]. In this paper we propose the following:

Theorem 1. Let $\Gamma \subset \mathbb{Q}^{*}$ be a finitely generated group of rank $r$ and let $m \in \mathbb{N}$. Then, as $x \rightarrow \infty$, uniformly in $m$,

$$
A_{\Gamma}(x, m)=\varrho_{\Gamma, m} \frac{x}{\log x}+O_{\Gamma}\left(\tau(m) m \times x\left(\frac{(\log \log x)^{2}}{\log x}\right)^{1+\frac{1}{3 r+3}}\right),
$$

where if $\gamma(f, t)=\prod_{\ell \mid f} \ell^{v_{\ell}(t)+1}$,

$$
\mathcal{S}_{m}=\{n \in \mathbb{N}: \operatorname{Rad}(n) \mid m \text { and } m \mid n\}
$$

and if $\mathbb{Q}\left(\zeta_{k}, \Gamma^{1 / h}\right)$ is the extension of $\mathbb{Q}$ generated by $\zeta_{k}=e^{2 \pi i / k}$ and by the $h-t h$ roots of all the elements of $\Gamma$, then

$$
\varrho_{\Gamma, m}=\sum_{n \in \mathcal{S}_{m}} \sum_{\substack{d|n \\ f| n}} \frac{\mu(d) \mu(f)}{\left[\mathbb{Q}\left(\zeta_{n d}, \Gamma^{1 / \gamma\left(f, \frac{n}{m}\right)}\right): \mathbb{Q}\right]} .
$$

In the case when $\Gamma \subset \mathbb{Q}^{+}$, the group of strictly positive rational numbers, we express $\varrho_{\Gamma, m}$ in terms of the orders of the groups

$$
\Gamma(t)=\Gamma \mathbb{Q}^{* t} / \mathbb{Q}^{* t} .
$$

Theorem 2. Assume that $\Gamma$ is a finitely generated subgroup of $\mathbb{Q}^{+}$and that $m \in \mathbb{N}$. For any squarefree integer $\eta$, let $t_{\eta}=\infty$ if either $m$ is odd or for all $t \geq 0$, $\eta^{2^{t}} \mathbb{Q}^{* 2^{t+1}} \notin \Gamma\left(2^{t+1}\right)$ and $t_{\eta}=\min \left\{t \in \mathbb{N}: \eta^{2^{t}} \mathbb{Q}^{* 2^{t+1}} \in \Gamma\left(2^{t+1}\right)\right\}$ otherwise. Furthermore, let $s_{\eta}=v_{2}\left(\frac{\delta(\eta)}{m}\right)$, where $\delta(\eta)$ is the discriminant of $\mathbb{Q}(\sqrt{\eta})$ and let $\sigma_{\Gamma}=\prod_{\ell \in \operatorname{Supp} \Gamma} \ell$. Then

$$
\varrho_{\Gamma, m}=\frac{1}{\varphi(m)} \prod_{\ell \mid m}\left(1-\sum_{j \geq 2} \frac{\ell-1}{\ell^{j}\left|\Gamma\left(\ell^{j}\right)\right|}\right)\left(1-\sum_{\eta \mid \operatorname{gcd}\left(m, \sigma_{\Gamma}\right)} \psi_{\eta}\right),
$$

where

$$
\psi_{\eta}= \begin{cases}0 & \text { if } t_{\eta}=\infty, \\ \sum_{k>t_{\eta}} \frac{1}{2^{k}\left|\Gamma\left(2^{k}\right)\right|} & \text { if } s_{\eta} \leq t_{\eta}<\infty, \\ -\frac{1}{2^{s_{\eta}}\left|\Gamma\left(2^{s_{\eta}}\right)\right|}+\sum_{k>s_{\eta}} \frac{1}{2^{k}\left|\Gamma\left(2^{k}\right)\right|} & \text { if } s_{\eta}>t_{\eta} .\end{cases}
$$

Remarks. (1) The condition $\Gamma \subset \mathbb{Q}^{+}$is not essential. It is mainly due to the fact that the group $\left(\Gamma \cap \mathbb{Q}\left(\zeta_{m}\right)^{* 2^{\alpha}}\right) \cdot \mathbb{Q}^{* 2^{\alpha}} / \mathbb{Q}^{* 2^{\alpha}}$ is easy to describe when $\Gamma \subset \mathbb{Q}^{+}$. This is done in Corollary 1. However, similar expressions for $\varrho_{\Gamma, m}$ as in Theorem 3 should be derived also for groups containing negative numbers and, in particular, containing -1 .

(2) It is plain that the Generalized Riemann Hypothesis for the Dedekind zeta functions of the fields $\mathbb{Q}\left(\zeta_{m}, \Gamma^{1 / d}\right)(d \mid m)$ allows a sharper error term in Theorem 1 In fact, applying the Generalized Riemann Hypothesis, and proceeding along the lines of the proof of Theorem 1 and applying 
[17. Lemma 5] rather than Lemma 4, it can be shown that, as $x \rightarrow \infty$, uniformly in $m$,

$$
A_{\Gamma}(x, m)=\varrho_{\Gamma, m} \operatorname{li}(x)+O_{\Gamma}\left(\tau(m)^{3} x^{3 / 4} \log x\right) .
$$

(3) All the series involved in the expression for $\varrho_{\Gamma, m}$ are convergent since they are bounded by geometric series. In the case when $\Gamma$ is finitely generated with rank $r$, for every prime power $\ell^{j}$, the following identity holds (see (5)):

$$
\left|\Gamma\left(\ell^{j}\right)\right|=\ell^{\max \left\{0, j-v_{\ell}\left(\Delta_{1}\right), \cdots,(r-1) j-v_{\ell}\left(\Delta_{r-1}\right), r j-v_{\ell}\left(\Delta_{r}\right)\right\}},
$$

where for $i=1, \ldots, r, \Delta_{i}$ is the $i$-th exponent of $\Gamma$ (defined in (44)). Therefore,

$$
\sum_{j>v_{\ell}\left(\Delta_{r}\right)} \frac{1}{\ell^{j}\left|\Gamma\left(\ell^{j}\right)\right|}=\ell^{v_{\ell}\left(\Delta_{r}\right)} \sum_{j>v_{\ell}\left(\Delta_{r}\right)} \frac{1}{\ell^{(r+1) j}}=\frac{\ell^{-r v_{\ell}\left(\Delta_{r}\right)}}{\ell^{r+1}-1} .
$$

This implies that $\varrho_{\Gamma, m} \in \mathbb{Q}^{+}$. Another immediate consequence of (1) is that if $\operatorname{gcd}\left(m, \Delta_{r-1}\right)=1$ and either $m$ is odd $\operatorname{or} \operatorname{gcd}\left(m, \sigma_{\Gamma}\right)=1$, then

$$
\varrho_{\Gamma, m}=\frac{1}{\varphi(m)} \prod_{\ell \mid m}\left(1-\frac{\ell-1}{\ell^{r}-1}\left[1-\frac{\ell^{r}(\ell-1)}{\ell^{r v_{\ell}\left(\Delta_{r}\right)}\left(\ell^{r+1}-1\right)}\right]\right) .
$$

(4) If one sets $\Delta_{0}=1$, then (2) holds also for $r=1$. More precisely, if $\Gamma=\langle a\rangle$, where $a \in \mathbb{Q}^{*} \backslash\{ \pm 1\}, a=b^{h}$ where $b$ is not the power on any rational number so that $h=\Delta_{1}$, we write (in a unique way) $b=a_{1} a_{2}^{2}$, where $a_{1}$ is a squarefree integer. Then

$$
1-\sum_{j \geq 1} \frac{\ell-1}{\ell^{j}\left|\frac{\left\langle b^{h}\right\rangle \mathbb{Q}^{*} \ell^{j}}{\mathbb{Q}^{\ell^{j}}}\right|}=\frac{1}{\ell^{v_{\ell}(h)}} \frac{\ell}{\ell+1} \text { and } \sum_{k>r_{a_{1}}} \frac{1}{2^{k}\left|\frac{\left\langle b^{h}\right\rangle \mathbb{Q}^{* 2^{k}}}{\mathbb{Q}^{* 2^{k}}}\right|}=\frac{1}{32^{v_{2}(h)}}
$$

since $r_{a_{1}}=v_{2}(h), r_{1}=0$ and since $s_{a_{1}}=v_{2}\left(\frac{\delta\left(a_{1}\right)}{m}\right)$. By Theorem 2 we obtain that $\varrho_{\left\langle b^{h}\right\rangle, m}$ equals:

$$
\frac{1}{m} \prod_{\ell \mid m} \frac{\ell^{2-v_{\ell}(h)}}{\ell^{2}-1} \times \begin{cases}\frac{1}{2} & \text { if }\left[2, a_{1}\right] \mid m \text { and } v_{2}\left(\delta\left(a_{1}\right)\right) \leq v_{2}(m h), \\ 1+\frac{1}{2^{2 v_{2}\left(\frac{\delta\left(a_{1}\right)}{h m}\right)}} & \text { if }\left[2, a_{1}\right] \mid m \text { and } v_{2}\left(\delta\left(a_{1}\right)\right)>v_{2}(m h), \\ 1 & \text { if }\left[2, a_{1}\right] \nmid m .\end{cases}
$$

This formula is consistent with the formula in [16, Theorem 1.3].

(5) An immediate consequence of the previous remark is that $\varrho_{\Gamma, m} \neq 0$ for any group $\Gamma$ and for any $m$. In fact, $\varrho_{\langle a\rangle, m}>0$ for any $a \in \mathbb{Q}^{*}$ and if $\Gamma^{\prime} \subset \mathbb{Q}^{*}$ is a subgroup with $\Gamma^{\prime} \subset \Gamma$, then $\operatorname{ord}_{p} \Gamma^{\prime} \mid \operatorname{ord}_{p} \Gamma$ for any prime $p \notin \operatorname{Supp} \Gamma$. Therefore, $\varrho_{\Gamma, m} \geq \varrho_{\Gamma^{\prime}, m}>0$.

(6) In the special case when $\Gamma=\left\langle d_{1}, d_{2}\right\rangle$ with $d_{1}, d_{2} \in \mathbb{Q}^{+}$, multiplicatively independent, we have that $\operatorname{rank} \Gamma=2$. So for $\ell \geq 3$,

$$
\Gamma\left(\ell^{j}\right)= \begin{cases}1 & \text { if } j \leq v_{\ell}\left(\Delta_{1}\right) \\ \ell^{j-v_{\ell}\left(\Delta_{1}\right)} & \text { if } v_{\ell}\left(\Delta_{1}\right)<j \leq v_{\ell}\left(\Delta_{2} / \Delta_{1}\right), \\ \ell^{2 j-v_{\ell}\left(\Delta_{2}\right)} & \text { if } j>v_{\ell}\left(\Delta_{2} / \Delta_{1}\right) .\end{cases}
$$

Hence

$1-\sum_{j \geq 1} \frac{\ell-1}{\ell^{j}\left|\Gamma\left(\ell^{j}\right)\right|}=\frac{1}{\ell^{v_{\ell}\left(\Delta_{1}\right)}} \cdot \frac{\ell}{\ell+1}+\frac{1}{\ell^{2 v_{\ell}\left(\Delta_{2} / \Delta_{1}\right)}} \cdot\left(\frac{\ell^{v_{\ell}\left(\Delta_{1}\right)}}{\ell+1}-\frac{1}{\ell^{2}+\ell+1}\right)$. 
This identity can be used in Theorem 3 to explicitly compute $\varrho_{\left\langle d, d_{2}\right\rangle, m}$ in the case when $m$ is odd or when $\operatorname{gcd}\left(m, \sigma_{\left\langle d, d_{2}\right\rangle}\right)=1$.

(7) If $\Gamma \subset \mathbb{Q}^{*}$ is the multiplicative subgroup generated by $r$ distinct prime numbers $p_{1}, \ldots, p_{r}$, then $\left|\Gamma\left(\ell^{j}\right)\right|=\ell^{r j}$ for all $j$, and if $\eta$ is a divisor of $\operatorname{gcd}\left(m, p_{1} \cdots p_{r}\right)$, then $t_{\eta}=0$. We deduce that

$$
\varrho_{\left\langle p_{1}, \cdots, p_{r}\right\rangle, m}=\frac{1}{\varphi(m)} \prod_{\substack{\ell \mid m, \ell>2}} \frac{\ell\left(\ell^{r}-1\right)}{\ell^{r+1}-1} \times\left(1-\frac{\psi}{2^{r+1}-1}\right),
$$

where $u_{k}=\#\left\{\eta \in \mathbb{N}: \eta \mid \operatorname{gcd}\left(m, p_{1} \cdots p_{r}\right), \eta \equiv k(\bmod 4)\right\}$

$$
\psi=\psi_{\left\langle p_{1}, p_{2}, \cdots, p_{r}\right\rangle, m}= \begin{cases}0 & \text { if } 2 \nmid m, \\ u_{1}+\left(\frac{1}{2^{r}}-1\right)\left[\frac{u_{2}}{2^{r+1}}+u_{3}\right] & \text { if } 2 \| m, \\ u_{1}+\left(\frac{1}{2^{r}}-1\right) u_{2}+u_{3} & \text { if } 4 \| m, \\ u_{1}+u_{2}+u_{3} & \text { if } 8 \mid m .\end{cases}
$$

Several computations of the densities $\varrho_{\left\langle p_{1}, \cdots, p_{r}\right\rangle, m}$ are presented in Section 8

(8) Among the various consequences of Theorem 1 one can also compute the density of the set of primes for which $\operatorname{ord}_{p} \Gamma$ is $k$-free (i.e., not divisible by the $k$-power of any prime). More precisely, if $k \geq 2$ and $\Gamma$ is finitely generated with rank $r$, then

$\#\left\{p \leq x: p \notin \operatorname{Supp} \Gamma, \operatorname{ord}_{p}(\Gamma)\right.$ is $k$-free $\}$

$$
=\left(\beta_{\Gamma, k}+O_{k, \Gamma}\left(\frac{(\log \log x)^{3}}{\log (k-1) /((k+1)(3 r+3))} x\right)\right) \frac{x}{\log x},
$$

where

$$
\beta_{\Gamma, k}=\sum_{m=1}^{\infty} \mu(m) \varrho_{\Gamma, m^{k}}
$$

In the special case when $\Gamma=\left\langle p_{1}, \cdots, p_{r}\right\rangle \subset \mathbb{Q}^{*}$, where $p_{j}$ is prime for all $j=1, \ldots, r$ and $p_{j}<p_{j+1}$ for all $j=1, \ldots, r-1$, we have that

$$
\beta_{\Gamma, k}=\beta_{r, k} \times \tilde{\beta}_{\Gamma, k},
$$

where

$$
\beta_{r, k}=\prod_{\ell>2}\left(1-\frac{\ell^{r}-1}{\ell^{k-2}(\ell-1)\left(\ell^{r+1}-1\right)}\right)
$$

and $\tilde{\beta}_{\Gamma, k} \in \mathbb{Q}^{+}$. Furthermore, if $k \geq 3$ or $p_{1} \geq 3$, then $\tilde{\beta}_{\Gamma, k}$ equals

$$
1-\frac{1}{2^{k-1}}\left[1-\frac{\operatorname{gcd}\left(2, p_{1}\right)}{2^{r+1}-1} \prod_{j=1}^{r}\left(1-\frac{p_{j}^{r}-1}{p_{j}^{k-2}\left(p_{j}-1\right)\left(p_{j}^{r+1}-1\right)-\left(p_{j}^{r}-1\right)}\right)\right],
$$

while, if $k=2$ and $p_{1}=2, \tilde{\beta}_{\Gamma, k}$ equals

$$
\frac{1}{2}+\frac{1}{2\left(2^{r+1}-1\right)} \prod_{j=1}^{r}\left(1-\frac{p_{j}^{r}-1}{\left(p_{j}-1\right)\left(p_{j}^{r+1}-1\right)-\left(p_{j}^{r}-1\right)}\right) .
$$


The proof of the above statement is carried out along the lines of [16,

Theorem 1.2]. Indeed, one starts from the identity

$$
\#\left\{p \leq x: p \notin \operatorname{Supp} \Gamma, \operatorname{ord}_{p}(\Gamma) \text { is } k \text {-free }\right\}=\sum_{m=1}^{\infty} \mu(m) A_{\Gamma}\left(x, m^{k}\right) .
$$

The mail term is obtained by applying Theorem 1 to the values of $m \leq$ $\log ^{1 /(2 k(3 r+3))} x$. For $\log ^{1 /(2 k(3 r+3))} x<m \leq \log ^{2} x$, one uses the bound $A_{\Gamma}\left(x, m^{k}\right) \leq \pi\left(x, m^{k}, 1\right)$ and the Brun-Titchmarch Theorem. We will omit further details.

As for most of the results regarding properties of the index and the order of subgroups of $\mathbb{F}_{p}^{*}$, the techniques are those of the pioneering work by C. Hooley [7], where Artin's Conjecture for primitive roots is established as one of the consequences of the Generalized Riemann Hypothesis.

The first to consider higher rank groups in relation to the Lang-Trotter Conjecture were Gupta and Ram Murty in [4. Their approach led to the quasi-resolution of the Artin's Conjecture by Gupta, Ram Murty and Heath-Brown [3, 6].

\section{Notational CONVEntions}

Throughout the paper, the letters $p$ and $\ell$ always denote prime numbers. As usual, we use $\pi(x)$ to denote the number of $p \leq x$ and

$$
\operatorname{li}(x)=\int_{2}^{x} \frac{d t}{\log t}
$$

denotes the logarithmic integral function.

$\varphi, \mu$ and $\tau$ are, respectively, the Euler, the Möbius and the number of divisors functions. An integer is said to be squarefree if it is not divisible for the square of any prime number and more generally it is said $k$-free if it is not divisible by the $k$-th power of any prime number.

For $n \in \mathbb{N}$, $\operatorname{Rad}(n)$ denotes the radical of $n$, the largest squarefree integer dividing $n$. For $\alpha \in \mathbb{Q}^{*}, v_{\ell}(\alpha)$ denotes the $\ell$-adic valuation of $\alpha$ and if $\eta \in \mathbb{Q}^{*}, \delta(\eta)$ denotes the field discriminant of $\mathbb{Q}(\sqrt{\eta})$. So, if

$$
\delta_{0}(\alpha)=\operatorname{sgn}(\alpha) \prod_{v_{\ell}(\alpha) \equiv 1 \bmod 2} \ell,
$$

then $\delta(\eta)=\delta_{0}(\eta)$ if $\delta_{0}(\eta) \equiv 1(\bmod 4)$ and $\delta(\eta)=4 \delta_{0}(\eta)$ otherwise.

For functions $F$ and $G>0$ the notations $F=O(G)$ and $F \ll G$ are equivalent to the assertion that the inequality $|F| \leq c G$ holds with some constant $c>0$. In what follows, all constants implied by the symbols $O$ and $\ll$ may depend (where obvious) on the small real parameter $\epsilon$ but are absolute otherwise; we write $O_{\rho}$ and $\ll_{\rho}$ to indicate that the implied constant depends on a given parameter $\rho$.

\section{Finitely generated subgroups of $\mathbb{Q}^{*}$}

Let $\Gamma$ be a finitely generated subgroup of $\mathbb{Q}^{*}$ of rank $r$ and let $\left(a_{1}, \ldots, a_{r}\right)$ be a $\mathbb{Z}$-basis of $\Gamma$. We write $\operatorname{Supp}(\Gamma)=\left\{p_{1}, \ldots, p_{s}\right\}$. Then we can construct the 
$s \times r$-matrix with coefficients in $\mathbb{Z}:$

$$
M\left(a_{1}, \ldots, a_{r}\right)=\left(\begin{array}{ccc}
\alpha_{1,1} & \cdots & \alpha_{1, r} \\
\vdots & & \vdots \\
\vdots & & \vdots \\
\alpha_{s, 1} & \cdots & \alpha_{s, r}
\end{array}\right),
$$

defined by the property that $\left|a_{i}\right|=p_{1}^{\alpha_{1, i}} \cdots p_{s}^{\alpha_{s, i}}$. It is clear that the rank of $\left.M\left(a_{1}, \ldots, a_{r}\right)\right)$ equals $r$. This of course implies $r \leq s$. For all $i=1, \ldots, r$, we define the $i$-th exponent of $\Gamma$ by

$$
\Delta_{i}=\Delta_{i}(\Gamma)=\operatorname{gcd}\left(\operatorname{det} A: A \text { is a } i \times i-\text { minor of } M\left(a_{1}, \ldots, a_{r}\right)\right) .
$$

So $\Delta_{i}$ is the nonnegative greatest common divisor of all the minors of size $i$ of $M\left(a_{1}, \ldots, a_{r}\right)$. We also set $\Delta_{k}=\Delta_{k}(\Gamma)=1$ for $k \leq 0$ and $\Delta_{k}=\Delta_{k}(\Gamma)=0$ for $k>r$. It can be shown (see [1, Section 3]) that $\Delta_{1}, \ldots, \Delta_{r}$ are well defined and do not depend on the choice of the basis $\left(a_{1}, \ldots, a_{r}\right)$ and on the ordering of the support $\left\{p_{1}, \ldots, p_{s}\right\}$. Furthermore, from the Dedekind formula expansion for determinants, we deduce that

$$
\Delta_{i} \Delta_{j} \mid \Delta_{i+j} \quad \forall i, j \geq 0 .
$$

For $m \in \mathbb{N}$, we have the following identity (see [1, Proposition 2, page 129 and the preceding pages])

$$
|\Gamma(m)|=\left|\Gamma \mathbb{Q}^{* m} / \mathbb{Q}^{* m}\right|=\frac{\varepsilon_{m, \Gamma} \times m^{r}}{\operatorname{gcd}\left(m^{r}, m^{(r-1)} \Delta_{1}, \ldots, m \Delta_{r-1}, \Delta_{r}\right)},
$$

where

$$
\varepsilon_{m, \Gamma}= \begin{cases}1 & \text { if } m \text { is odd or if }-1 \notin \Gamma \mathbb{Q}^{* m} \\ 2 & \text { if } m \text { is even and }-1 \in \Gamma \mathbb{Q}^{* m}\end{cases}
$$

Finally, from (5) and (6), we deduce the bounds

$$
2 m^{r} \geq|\Gamma(m)| \geq \frac{m^{r}}{\Delta_{r}(\Gamma)} .
$$

\section{Locally finite Subgroups of $\mathbb{Q}^{*}$}

The case when $\Gamma$ is not finitely generated is also of interest. In order to apply the machinery used for finitely generated groups, we shall make some necessary assumptions. We say that $\Gamma$ has thin support if $\operatorname{Supp} \Gamma$ has 0 density in the set of prime numbers. This hypothesis assures that $\operatorname{ord}_{p}(\Gamma)$ is defined for almost all primes $p$. Furthermore, we say that $\Gamma$ is locally finite if $\Gamma(m)=\Gamma \mathbb{Q}^{* m} / \mathbb{Q}^{* m}$ is finite for every $m \in \mathbb{N}$.

If $\Gamma$ is locally finite, we know that the exponent of finite group $\Gamma(m)$ is a divisor of $m$. We denote by $r_{\Gamma}(m)$ the finite group rank of $\Gamma(m)$. This means that

$$
\Gamma(m) \cong \frac{\mathbb{Z}}{m_{1} \mathbb{Z}} \oplus \cdots \oplus \frac{\mathbb{Z}}{m_{r} \mathbb{Z}}
$$

where $r=r_{\Gamma}(m), m_{1}\left|m_{2}\right| \cdots\left|m_{r}\right| m, m_{1}>1$. If $\eta_{1} \mathbb{Q}^{* m}, \ldots, \eta_{r_{\Gamma(m)}} \mathbb{Q}^{* m}$ is a set of generators for $\Gamma(m)$, we define the $m$-th local support as

$$
\operatorname{Supp}_{m} \Gamma=\left\{p \in \operatorname{Supp} \Gamma: v_{p}\left(\eta_{j}\right) \neq 0, \text { for some } j=1, \ldots, r_{\Gamma(m)}\right\} \text {. }
$$


and

$$
\sigma_{\Gamma, m}=\prod_{p \in \operatorname{Supp}_{m} \Gamma} p
$$

Furthermore, it is easy to check that

$$
\Gamma(m)=\left\langle\eta_{1}, \ldots, \eta_{r_{\Gamma(m)}}\right\rangle \mathbb{Q}^{* m} / \mathbb{Q}^{* m} .
$$

So we can apply the identity of (5) obtaining

$$
|\Gamma(m)|=\frac{\varepsilon_{m, \Gamma} \times m^{r_{\Gamma(m)}}}{\operatorname{gcd}\left(m^{r_{\Gamma(m)}}, m^{r_{\Gamma(m)}-1} \Delta_{1}(\tilde{\Gamma}), \ldots, m \Delta_{r_{\Gamma(m)}-1}(\tilde{\Gamma}), \Delta_{r_{\Gamma(m)}}(\tilde{\Gamma})\right)},
$$

where $\tilde{\Gamma}=\left\langle\eta_{1} \ldots \eta_{r_{\Gamma(m)}}\right\rangle$ and $\varepsilon_{m, \Gamma}$ is defined in (6) .

The free subgroup of $\mathbb{Q}^{*}$ generated by any fixed set of primes $S$ with zero density is a thin support subgroup. However, if $S$ is infinite, such subgroup is not locally finite. Here we consider the following family of locally finite, thin support, not finitely generated subgroups of $\mathbb{Q}^{*}$ :

Definition 1. Let $S$ be a set of primes with 0 density and write

$$
S=\left\{p_{1}, p_{2}, \cdots\right\},
$$

where $p_{i} \leq p_{i+1}$ for all $i \in \mathbb{N}$. Let $\Gamma_{S}$ be the subgroup of $\mathbb{Q}^{*}$ generated by the $k !-$ powers of the $p_{k}$ 's. That is,

$$
\Gamma_{S}=\left\langle p_{1}, p_{2}^{2 !}, \ldots, p_{k}^{k !}, \ldots\right\rangle .
$$

It is plain that $\Gamma_{S}$ is a free $\mathbb{Z}$-module of infinite rank. Furthermore, $S=\operatorname{Supp} \Gamma_{S}$ so that $\Gamma_{S}$ has thin support. However, for every $m \in \mathbb{N}$, we have the identity:

$$
\Gamma_{S}(m)=\frac{\Gamma_{S} \mathbb{Q}^{* m}}{\mathbb{Q}^{* m}}=\frac{\left\langle p_{1}, p_{2}^{2 !}, \ldots, p_{m-1}^{(m-1) !}\right\rangle \mathbb{Q}^{* m}}{\mathbb{Q}^{* m}}
$$

Hence

Proposition 1. Let $m \in \mathbb{N}$ and let $S$ be a set of prime numbers. Then $\Gamma_{S}$ is locally finite and satisfies the following properties:

(1) $r_{\Gamma_{S}(m)}=r(m)=\max \{k \in \mathbb{N}: m \nmid k !\} \leq m-1$;

(2) if $\ell$ is prime, then $r\left(\ell^{\alpha}\right) \leq \alpha \ell-1$;

(3) $r\left(\ell^{\alpha}\right)=\alpha \ell-1$ for $\alpha \leq \ell$;

(4) $\# \Gamma_{S}(m)=\prod_{j \leq r(m)} \frac{m}{\operatorname{gcd}(m, j !)}$ is a multiplicative function;

(5) $\operatorname{Supp}_{m} \Gamma_{S}=\left\{p_{1}, \ldots, p_{r_{\Gamma_{S}(m)}}\right\} \subset\left\{p_{1}, \ldots, p_{m-1}\right\}$.

Proof. The first statement is clear from the definition and, for the second, observe that $v_{\ell}((\alpha \ell) !)$ satisfies

$$
v_{\ell}((\alpha \ell) !)=\alpha+\sum_{j \geq 1}\left[\frac{\alpha}{\ell^{j}}\right] \geq \alpha .
$$

This observation also implies that $r\left(\ell^{\alpha}\right)=\alpha \ell-1$ for $\alpha \leq \ell$. As for the fourth statement, it is enough to observe that

$$
\frac{\Gamma_{S} \mathbb{Q}^{* m}}{\mathbb{Q}^{* m}} \cong \bigoplus_{j=1}^{\infty} \frac{\left\langle p_{j}^{j !}\right\rangle \mathbb{Q}^{* m}}{\mathbb{Q}^{* m}}
$$


and to apply the fact that

$$
\# \frac{\left\langle p_{j}^{j !}\right\rangle \mathbb{Q}^{* m}}{\mathbb{Q}^{* m}}=\frac{m}{\operatorname{gcd}(m, j !)}
$$

is a multiplicative function of $m$ which is identically 1 if $j>r(m)$. The last statement is also clear from the definition of $\operatorname{Supp}_{m} \Gamma_{S}$.

Theorem 3. Let $S$ be a set of prime numbers with 0 density and let $m \in \mathbb{N}$ be either an odd number or such that $\operatorname{gcd}\left(m, \sigma_{\Gamma_{S}, m}\right)=1$. Then, as $x \rightarrow \infty$,

$$
A_{\Gamma_{S}}(x, m) \sim \frac{\chi_{\Gamma_{S}, m}}{\varphi(m)} \cdot \frac{x}{\log x},
$$

where

$$
\chi_{\Gamma_{S}, m}=\prod_{\ell \mid m}\left(1-\sum_{\alpha \geq 1} \frac{\ell-1}{\ell^{\alpha+\sum_{j \geq 1} \max \left\{0, \alpha-v_{\ell}(j !)\right\}}}\right) .
$$

We will omit the proof of Theorem 3 since it is similar to the proof of Theorem 1 , where the main ingredient Lemma 4 is replaced with Lemma 5.

Remarks. (1) When $\Gamma$ is not finitely generated, the rationality of $\varrho_{\Gamma, m}$ does not hold in general. In fact, if $\ell$ is an odd prime, $\Gamma=\left\langle p_{1}, p_{2}^{a_{2}}, \ldots, p_{k}^{a_{k}}, \ldots\right\rangle$ where $\left\{p_{1}, p_{2} \ldots\right\}$ is a zero density set of primes and $a_{k}=\ell^{\beta_{k}} k ! / \ell^{v_{\ell}(k !)}$ where $\beta_{1}=0$ and for $k \geq 2, \beta_{k}$ is defined by

$$
\beta_{k}=j \text { if and only if } j !-j<k \leq(j+1) !-j-1,
$$

then $\Gamma$ has thin support and it is locally finite. Furthermore,

$$
\Gamma\left(\ell^{j}\right)=\ell^{\max \left\{k \in \mathbb{N}: \beta_{k}<j\right\}}=\ell^{j !-j} .
$$

Hence

$$
\varrho_{\Gamma, \ell}=1-(\ell-1) \sum_{j \geq 1} \frac{1}{\ell^{j !}}
$$

is rationally dependent to the Liouville transcendental number.

(2) The conditions that either $m$ is odd or that $\operatorname{gcd}\left(m, \sigma_{\Gamma_{S}, m}\right)=1$ in the statement of Theorem 3 can be removed at the cost of complicating the expression for $\chi_{\Gamma_{S}, m}$.

(3) It was proven in [1] that if $\Gamma \subset \mathbb{Q}^{*}$ is a finitely generated subgroup, the Generalized Riemann Hypothesis implies that the set of primes for which $\operatorname{ind}_{p}(\Gamma)=1$ has a density $\delta_{\Gamma}$ that equals

$$
\prod_{\ell>2}\left(1-\frac{1}{|\Gamma(\ell)|(\ell-1)}\right)\left(1-\frac{1}{|\Gamma(2)|} \sum_{\substack{\xi \in \Gamma(2) \\ \xi \equiv 1 \bmod 4}} \prod_{\ell \mid \xi} \frac{1}{1-|\Gamma(\ell)|(\ell-1)}\right) .
$$

This formula also holds for thin support, locally finite subgroups. In particular, if $S=\left\{p_{1}, p_{2}, \ldots\right\}$ is a set of prime numbers with zero density, then

$$
\Gamma_{S}(\ell)=\frac{\left\langle p_{1}, p_{2}^{2 !}, \ldots, p_{\ell-1}^{(\ell-1) !}\right\rangle \mathbb{Q}^{* \ell}}{\mathbb{Q}^{* \ell}}
$$


and $\left|\Gamma_{S}(\ell)\right|=\ell^{r(\ell)}=\ell^{\ell-1}$ by (3) in Proposition 1, Therefore,

$$
\delta_{\Gamma_{S}}=\prod_{\ell}\left(1-\frac{1}{\ell^{\ell-1}(\ell-1)}\right) \times\left(1+\tau_{p_{1}}\right),
$$

where

$$
\tau_{p_{1}}= \begin{cases}\frac{1}{p_{1}^{p_{1}-1}\left(p_{1}-1\right)-1} & \text { if } p_{1} \equiv 1 \bmod 4, \\ 0 & \text { otherwise. }\end{cases}
$$

Example. Let $\mathbb{G}=\{3,5,11,17,29, \ldots\}$ denote the set of (youngest) twin primes which is well known to have density 0 and we will also assume to be infinite. Hence

$$
\Gamma_{\mathbb{G}}=\left\langle 3,5^{2}, 11^{6}, 17^{24}, 29^{120}, \ldots\right\rangle .
$$

In the following table we compare:

- the values of $\varrho_{\Gamma_{\mathbb{G}}, m}\left(1^{\text {st }}\right.$ row $)$;

- the values of $\frac{A_{\Gamma_{\mathbb{G}}, m}\left(10^{6}, 3\right)}{\pi\left(10^{6}\right)}\left(2^{\text {nd }}\right.$ row $)$;

- the values of $\frac{A_{\Gamma_{\mathbb{G}}, m}\left(10^{6}, 3\right)}{\#\left\{p \leq 10^{6}: p \notin \mathbb{G}\right\}}\left(3^{\text {rd }}\right.$ row $)$

for $m=2, \ldots, 13$. Note that the numbers are truncated (not approximated) to the ninth decimal digit.

\begin{tabular}{|c|c|c|c|c|c|c|}
\hline$m$ & 2 & 3 & 4 & 5 & 6 & 7 \\
\hline & 0.733383118 & 0.462912155 & 0.366691559 & 0.249679999 & 0.447527842 & 0.166665452 \\
& 0.681724375 & 0.462725165 & 0.314364697 & 0.214757063 & 0.447743891 & 0.145086499 \\
& 0.760844529 & 0.516428520 & 0.350849505 & 0.239681524 & 0.499708537 & 0.161925072 \\
\hline \hline$m$ & 8 & 9 & 10 & 11 & 12 & 13 \\
\hline & 0.183345779 & 0.154304051 & 0.178962194 & 0.099999999 & 0.108035882 & 0.083333333 \\
& 0.156959413 & 0.154564447 & 0.161800300 & 0.088397156 & 0.107302096 & 0.074052842 \\
& 0.175175943 & 0.172503021 & 0.180578659 & 0.098656429 & 0.119755456 & 0.082647330 \\
\hline
\end{tabular}

Finally, $\delta_{\Gamma_{\mathbb{G}}}=0.47203266462865646291 \cdots$ while

$$
\frac{\left|\left\{p \leq 10^{6}: p \notin \mathbb{G}, \operatorname{ind}_{p}\left(\Gamma_{\mathbb{G}}\right)=1\right\}\right|}{\pi\left(10^{6}\right)}=\frac{33059}{78498}=0.4211444878 \cdots
$$

and

$$
\frac{\left|\left\{p \leq 10^{6}: p \notin \mathbb{G}, \operatorname{ind}_{p}\left(\Gamma_{\mathbb{G}}\right)=1\right\}\right|}{\left|\left\{p \leq 10^{6}: p \notin \mathbb{G}\right\}\right|}=\frac{33059}{70335}=0.4700220374 \cdots
$$

5. The DEGREe $\left[\mathbb{Q}\left(\zeta_{m}, \Gamma^{1 / d}\right): \mathbb{Q}\right]$.

Let $\Gamma \subset \mathbb{Q}^{*}$ be a locally finite subgroup and let $m$ and $d$ be positive integers with $d \mid m$. We denote by $K_{m}$ the $m$-th cyclotomic field. So $K_{m}=\mathbb{Q}\left(\zeta_{m}\right)$, where $\zeta_{m}=e^{2 \pi i / m}$ is the primitive $m$-th root of unity. Furthermore, we denote $K_{m}\left(\Gamma^{1 / d}\right)$ the subfield of $\mathbb{C}$ generated over $K_{m}$ by the $d$-th roots of all elements of $\Gamma$. It is well known that $K_{m}\left(\Gamma^{1 / d}\right)$ is a finite Galois extension of $\mathbb{Q}$ and that there is an isomorphism

$$
\operatorname{Gal}\left(K_{m}\left(\Gamma^{1 / d}\right) / K_{m}\right) \cong \Gamma\left(K_{m}^{*}\right)^{d} /\left(K_{m}^{*}\right)^{d} .
$$

Details on the theory of Kummer's extensions can be found in Lang's book [10, Theorem 8.1]. The goal of this section is to prove the following: 
Lemma 1. Let $\Gamma \subset \mathbb{Q}^{*}$ be a locally finite subgroup. Let $m$ and $d$ be positive integers with $d \mid m$, set $\alpha=v_{2}(d)$ to be the 2 -adic valuation and let $k_{m, d}(\Gamma)$ denote the degree of the extension $K_{m}\left(\Gamma^{1 / d}\right) / \mathbb{Q}$. Then the degree

$$
k_{m, d}(\Gamma)=\frac{\varphi(m) \times|\Gamma(d)|}{\left|\mathcal{H}_{m, \alpha}\right|},
$$

where

$$
\mathcal{H}_{m, \alpha}=\left(\Gamma \cap K_{m}^{* 2^{\alpha}}\right) \mathbb{Q}^{* 2^{\alpha}} / \mathbb{Q}^{* 2^{\alpha}} .
$$

It is clear that if $d$ is odd, so that $\alpha=0$, then $\left|\mathcal{H}_{m, 0}\right|=1$. In the following statement we will describe explicitly $\mathcal{H}_{m, \alpha}$ in the case when $\Gamma$ contains only positive numbers.

Corollary 1. Given the Hypothesis of Lemma 1 , also assume that $\Gamma \subset \mathbb{Q}^{+}$and that $d$ is even so that $\alpha>0$. Then

$$
\mathcal{H}_{m, \alpha}=\left\{\eta \in \mathbb{N}: \eta\left|\operatorname{gcd}\left(m, \sigma_{\Gamma, m}\right), \eta^{2^{\alpha-1}} \cdot \mathbb{Q}^{* 2^{\alpha}} \in \Gamma\left(2^{\alpha}\right), \delta(\eta)\right| m\right\} .
$$

Proof of Corollary 1. First note that if $\zeta \in \Gamma$, then $\zeta \in K_{m}^{*} 2^{\alpha}$ if and only if $\sqrt[2 \alpha]{\zeta} \in$ $K_{m}^{*}$. Since, for $\zeta>0, \mathbb{Q}\left[\sqrt[2^{\alpha}]{\zeta}\right]$ is a Galois extension of $\mathbb{Q}$ only if its degree over $\mathbb{Q}$ is less than or equal to 2 , we deduce that $\zeta \cdot \mathbb{Q}^{* 2^{\alpha}}=\eta^{2^{\alpha-1}} \cdot \mathbb{Q}^{* 2^{\alpha}}$ for a unique squarefree $\eta \in \mathbb{N}$. Furthermore, $\mathbb{Q}(\sqrt{\eta}) \subset K_{m}$ if and only if $\delta(\eta) \mid m$ (see, for example, Weiss [19, page 264]). Finally, the conditions $\delta(\eta) \mid m$ and $\eta$ squarefree imply in particular that $\eta \mid \operatorname{Rad}(m)$ and this completes the proof.

Proof of Lemma 1. By the multiplicative property of the degree, we have that

$$
k_{m, d}(\Gamma)=\left[K_{m}\left(\Gamma^{1 / d}\right): \mathbb{Q}\right]=\varphi(m) \times\left|\operatorname{Gal}\left(K_{m}\left(\Gamma^{1 / d}\right) / K_{m}\right)\right| .
$$

By (8), since $\Gamma\left(K_{m}^{*}\right)^{d} /\left(K_{m}^{*}\right)^{d}$ is an abelian torsion group with exponent dividing $d$, we have that

$$
k_{m, d}(\Gamma)=\varphi(m) \prod_{\substack{\ell \text { prime } \\ \ell^{\alpha} \| d}}\left[K_{m}\left(\Gamma^{1 / \ell^{\alpha}}\right): K_{m}\right]=\varphi(m) \prod_{\substack{\ell \text { prime } \\ \ell^{\alpha} \| d}}\left|\Gamma K_{m}^{* \ell^{\alpha}} / K_{m}^{*} \ell^{\alpha}\right| .
$$

Now we apply the standard Isomorphism Theorems of finite groups and obtain that:

$$
\frac{\Gamma K_{m}^{* \ell^{\alpha}}}{K_{m}^{* \ell^{\alpha}}} \cong \frac{\Gamma}{\Gamma \cap K_{m}^{* \ell^{\alpha}}} \cong \frac{\Gamma \mathbb{Q}^{* \ell^{\alpha}} / \mathbb{Q}^{* \ell^{\alpha}}}{\left(\Gamma \cap K_{m}^{* \ell^{\alpha}}\right) \mathbb{Q}^{* \ell^{\alpha}} / \mathbb{Q}^{* \ell^{\alpha}}}
$$

If $\ell$ is odd, then $\Gamma \cap K_{m}^{*} \ell^{\alpha}=\Gamma \cap \mathbb{Q}^{* \ell^{\alpha}}$. Therefore,

$$
k_{m, d}(\Gamma)=\frac{\varphi(m)}{\left|\mathcal{H}_{m, v_{2}(d)}\right|} \times \prod_{\substack{\ell \text { prime } \\ \ell^{\alpha} \| d}}\left|\Gamma\left(\ell^{\alpha}\right)\right|=\frac{\varphi(m)}{\left|\mathcal{H}_{m, v_{2}(d)}\right|} \times|\Gamma(d)|,
$$

where $\mathcal{H}_{m, \alpha}=\left(\Gamma \cap K_{m}^{*} 2^{\alpha}\right) \mathbb{Q}^{* 2^{\alpha}} / \mathbb{Q}^{* 2^{\alpha}}$ and this concludes the proof.

\section{Chebotarev Density Theorem for $\mathbb{Q}\left(\zeta_{m}, \Gamma^{1 / d}\right)$}

In this section we apply the celebrated Chebotarev Density Theorem to the fields $\mathbb{Q}\left(\zeta_{m}, \Gamma^{1 / d}\right)$. We start by stating the result proven in [9] which, for simplicity, we specialize to the case of extensions of $\mathbb{Q}$ and trivial conjugacy classes: 
Lemma 2 (Effective, "unconditional" Chebotarev Density Theorem). Assume that $L / \mathbb{Q}$ is a Galois extension and denote by $n_{L}$ and $d_{L}$ the degree and the discriminant of $L$. Then there exist constants $c_{1}$ and $c_{2}$ such that if

$$
\log x>10 n_{L} \log ^{2} d_{L},
$$

then

$$
\#\left\{p \leq x: p \nmid d_{L}, p \text { split totally in } L / \mathbb{Q}\right\}=\frac{\operatorname{li}(x)}{n_{L}}+O\left(\frac{\operatorname{li}\left(x^{\beta_{0}}\right)}{n_{L}}+\frac{x}{e^{c_{1} \sqrt{\frac{\log x}{n_{L}}}}}\right)
$$

and $\beta_{0} \geq \frac{1}{2}$ satisfies

$$
\beta_{0} \leq \max \left\{1-\frac{1}{4 \log d_{L}}, 1-\frac{1}{c_{2} d_{L}^{1 / n_{L}}}\right\} .
$$

In order to apply the above result, we need a sufficiently sharp estimate for $\log d_{L}$. An adequate one can be found in [18].

Lemma 3. Assume that $L / \mathbb{Q}$ is a Galois extension and denote by $n_{L}$ and $d_{L}$ the degree and the discriminant of $L$. Then

$$
\frac{n_{L}}{2} \log \left(\operatorname{Rad}\left(d_{E}\right)\right) \leq \log d_{L} \leq\left(n_{L}-1\right) \log \left(\operatorname{Rad}\left(d_{E}\right)\right)+n_{L} \log n_{L} .
$$

Consider the Galois extension $\mathbb{Q}\left(\zeta_{m}, \Gamma^{1 / d}\right)$, where $d \mid m$ and where $\Gamma \subset \mathbb{Q}^{*}$ is a locally finite subgroup. So, by Lemma 1 ,

$$
n_{\mathbb{Q}\left(\zeta_{m}, \Gamma^{1 / d}\right)}=k_{m, d}(\Gamma) \leq m|\Gamma(d)| .
$$

Also note that the primes that ramify in such an extension are exactly those that either divide $m$ or those in $\operatorname{Supp}_{d} \Gamma$. Therefore, $\operatorname{Rad}\left(d_{\mathbb{Q}\left(\zeta_{m}, \Gamma^{1 / d}\right)}\right)=\operatorname{lcm}\left(\operatorname{Rad}(m), \sigma_{\Gamma, d}\right)$ and, by Lemma 3 ,

$$
\log \left(d_{\mathbb{Q}\left(\zeta_{m}, \Gamma^{1 / d}\right)}\right) \leq 2 m|\Gamma(d)| \log \left(m|\Gamma(d)| \sigma_{\Gamma, m}\right) .
$$

The conditions of uniformity of Lemma 2 are satisfied if

$$
(m|\Gamma(d)|)^{3} \log ^{2}\left(m|\Gamma(d)| \sigma_{\Gamma, m}\right) \leq c \log x
$$

for some $c>0$. We set $\pi_{\Gamma}(x, n, d)$ to be the number of primes up to $x$ that are unramified and split completely in $K_{n}\left(\Gamma^{1 / d}\right)$.

If we specialize the previous discussion to the case when $\Gamma$ is a finitely generated group and we use the upper bound in (7), we obtain:

Lemma 4. Assume that $\Gamma \subset \mathbb{Q}^{*}$ is a fixed finitely generated subgroup of rank $r$. Let $m, d \in \mathbb{N}$ be integers such that $d \mid m$. Then there exists constants $c_{1}$ and $c_{2}$ depending only on $\Gamma$ such that, uniformly for

$$
m \leq c_{1}\left(\frac{\log x}{(\log \log x)^{2}}\right)^{1 /(3 r+3)}
$$

as $x \rightarrow \infty$,

$$
\pi_{\Gamma}(x, m, d)=\frac{1}{k_{m, d}(\Gamma)} \operatorname{li}(x)+O_{\Gamma}\left(\frac{x}{e^{c_{2}} \sqrt[6]{\log x} \cdot \sqrt[3]{\log \log x}}\right) .
$$

If we specialize the previous discussion to the case when $\Gamma=\Gamma_{S}$, where $S$ is a set of primes with zero density, we obtain: 
Lemma 5. Let $S$ be a set of prime numbers with density zero. Let $m, d \in \mathbb{N}$ be integers such that $d \mid m$. Assume also that $\log \sigma_{\Gamma, m} \leq m^{m}$. Then, there exist absolute positive constants $c_{1}$ and $c_{2}<1$ such that for $x \rightarrow \infty$, uniformly for

$$
m \leq c_{1} \frac{\log \log x}{\log \log \log x}
$$

we have

$$
\pi_{\Gamma_{S}}(x, m, d)=\frac{1}{k_{m, d}\left(\Gamma_{S}\right)} \operatorname{li}(x)+O\left(x \exp \left(-(\log x)^{c_{2}}\right)\right.
$$

\section{Proofs of Theorems 1 and 2}

It is a criterion due to Dedekind that an odd prime $p \notin \operatorname{Supp} \Gamma$ splits totally in $K_{n}\left(\Gamma^{1 / d}\right)$ if and only $d$ divides the index $\operatorname{ind}_{p}(\Gamma)$ and $p \equiv 1(\bmod n)$. Therefore,

$$
\pi_{\Gamma}(x, n, d)=\#\left\{p \leq x: p \notin \operatorname{Supp} \Gamma, p \equiv 1(\bmod n), d \mid \operatorname{ind}_{p}(\Gamma)\right\} .
$$

The following combinatorial identity allows us to apply the Chebotarev Density Theorem.

Lemma 6. Let $m \in \mathbb{Z}$ and $\Gamma \leq \mathbb{Q}^{*}$. We have the identity

$$
A_{\Gamma}(x, m)=\sum_{n \in \mathcal{S}_{m}} \sum_{d \mid n} \sum_{f \mid m} \mu(d) \mu(f) \pi_{\Gamma}(x, n d, \gamma(f, n / m)),
$$

where

$$
\mathcal{S}_{m}=\{n \in \mathbb{N}: \operatorname{Rad}(n) \mid m \text { and } m \mid n\}
$$

and

$$
\gamma(f, k)=\prod_{\ell \mid f} \ell^{v_{\ell}(k)+1}
$$

Note that with the notation above, $\gamma(f, n / m) \mid n d$. In fact, for every $\ell \mid f$, $v_{\ell}(n)-v_{\ell}(m)+1 \leq v_{\ell}(n)+v_{\ell}(d)$ since $v_{\ell}(m) \geq 1$.

Proof. Let $p$ be a prime such that $p \notin \operatorname{Supp} \Gamma$ and $m \mid \operatorname{ord}_{p}(\Gamma)$. Then $m \mid p-1$ and there exists a unique $n \in \mathcal{S}_{m}$ such that $p \equiv 1 \bmod n$ and $\left(\frac{p-1}{n}, m\right)=1$ (indeed $\left.n=\prod_{\ell \mid m} \ell^{v_{\ell}(p-1)}\right)$. Hence

$$
\operatorname{ber} A_{\Gamma}(x, m)=\sum_{n \in \mathcal{S}_{m}} B_{\Gamma}(x, m)
$$

where $B_{\Gamma}(x, m)$ equals

$$
\#\left\{p \leq x: p \notin \operatorname{Supp} \Gamma, m \mid \operatorname{ord}_{p}(\Gamma), p \equiv 1(\bmod n),\left(\frac{p-1}{n}, m\right)=1\right\} .
$$

Now note that if $p$ is a prime with $p \notin \operatorname{Supp} \Gamma, p \equiv 1(\bmod n)$ and $\left(\frac{p-1}{n}, m\right)=1$, then

$$
\left.m \mid \operatorname{ord}_{p}(\Gamma) \quad \Longleftrightarrow \quad \operatorname{ind}_{p}(\Gamma), n\right) \mid \frac{n}{m} .
$$

Indeed, from the hypothesis that $n \in \mathcal{S}_{m}$ and from

$$
n=(p-1, n)=\left(\operatorname{ind}_{p}(\Gamma), n\right)\left(\operatorname{ord}_{p}(\Gamma), n\right)
$$


we deduce that $m \mid \operatorname{ord}_{p}(\Gamma)$ if and only if $m \mid\left(\operatorname{ord}_{p}(\Gamma), n\right)$ i.e., $\left(\operatorname{ind}_{p}(a), n\right) \mid \frac{n}{m}$. So we can rewrite $B_{\Gamma}(x, m)$ in $(\underline{10})$ as

$$
\#\left\{p \leq x: p \notin \operatorname{Supp} \Gamma,\left(\operatorname{ind}_{p}(\Gamma), n\right) \mid \frac{n}{m}, \quad p \equiv 1(\bmod n),\left(\frac{p-1}{n}, m\right)=1\right\} .
$$

Next we apply the inclusion-exclusion formula to the conditions $p \equiv 1(\bmod n)$ and $\left(\frac{p-1}{n}, m\right)=1$, so that $A_{\Gamma}(x, m)$ equals

$$
\sum_{n \in \mathcal{S}_{m}} \sum_{d \mid m} \mu(d) \#\left\{p \leq x: p \notin \operatorname{Supp} \Gamma,\left(\operatorname{ind}_{p}(\Gamma), n\right) \mid \frac{n}{m}, p \equiv 1(\bmod n d)\right\} .
$$

Finally, observe that, if $\gamma(f, n / m)$ is the quantity defined in the statement of the lemma, then

$$
\sum_{\substack{f\left|n \\ \gamma\left(f, \frac{n}{m}\right)\right| \operatorname{ind}_{p}(\Gamma)}} \mu(f)=\prod_{\substack{\left.\ell \mid n \\ v_{\ell}\left(\frac{n}{m}\right)<v_{\ell}\left(\operatorname{ind}_{p}(\Gamma)\right)\right)}}(1+\mu(\ell))= \begin{cases}1 & \text { if }\left(\operatorname{ind}_{p}(\Gamma), n\right) \mid \frac{n}{m}, \\ 0 & \text { otherwise. }\end{cases}
$$

So $A_{\Gamma}(x, m)$ equals

$$
\sum_{n \in \mathcal{S}_{m}} \sum_{\substack{d|m \\ f| n}} \mu(d) \mu(f) \#\left\{p \leq x: p \notin \operatorname{Supp} \Gamma, \gamma\left(f, \frac{n}{m}\right) \mid \operatorname{ind}_{p}(\Gamma), p \equiv 1(\bmod n d)\right\} .
$$

Applying the definition in (9) and the fact that $n$ and $m$ have the same radical, we deduce the claim.

Proof of Theorem 1. Let us start from the identity of Lemma 6 and rewrite it as:

$$
\begin{aligned}
A_{\Gamma}(x, m)= & \sum_{\substack{n \in \mathcal{S}_{m}, y \\
n m \leq y}} \sum_{\substack{d|n \\
f| n}} \mu(d) \mu(f) \pi_{\Gamma}\left(x, n d, \gamma\left(f, \frac{n}{m}\right)\right) \\
& +O\left(\sum_{\substack{n \in \mathcal{S}_{m}, d \mid m \\
n m>y}} \sum_{\Gamma \mid n} \pi_{\Gamma}\left(x, n d, \gamma\left(f, \frac{n}{m}\right)\right)\right) \\
= & \Sigma_{1}+O\left(\Sigma_{2}\right) .
\end{aligned}
$$

Note that Lemma 4 implies that if $y=c_{1}\left(\log x / \log ^{2} \log x\right)^{1 /(3 r+3)}$, then

$$
\begin{aligned}
\Sigma_{1} & =\sum_{\substack{n \in \mathcal{S}_{m}, n m \leq y}} \sum_{f \mid n} \mu(d) \mu(f) \pi_{\Gamma}\left(x, n d, \gamma\left(f, \frac{n}{m}\right)\right) \\
& =\sum_{\substack{n \in \mathcal{S}_{m}, n m \leq y}} \sum_{d \mid m}\left(\frac{\mu(d) \mu(f) \operatorname{li}(x)}{k_{d n, \gamma\left(f, \frac{n}{m}\right)}(\Gamma)}+O_{\Gamma}\left(\frac{x}{e^{c_{2}} \sqrt[6]{\log x} \cdot \sqrt[3]{\log \log x}}\right)\right) \\
& =\varrho_{\Gamma, m} \operatorname{li}(x)+E(x, y, m),
\end{aligned}
$$


where

$$
\begin{aligned}
E(x, y, m) & \ll \sum_{\substack{n \in \mathcal{S}_{m}, n m \leq y}} \frac{\tau(n) \tau(m) x}{e^{c_{2}} \sqrt[6]{\log x} \cdot \sqrt[3]{\log \log x}}+\sum_{\substack{n \in \mathcal{S}_{m}, n m>y}} \sum_{\substack{d|m \\
f| n}} \frac{\mu^{2}(d) \mu^{2}(f)}{k_{d n, \gamma(f, n / m)}(\Gamma)} \operatorname{li}(x) \\
& \ll \frac{\tau(m)}{m} \frac{x y \log y}{e^{c_{2}} \sqrt[6]{\log x} \cdot \sqrt[3]{\log \log x}}+\tau(m) \frac{m}{\varphi(m)} \frac{x}{\log x} \sum_{\substack{n \in \mathcal{S}_{m}, n>y / m}} \frac{1}{\varphi(n)},
\end{aligned}
$$

since $k_{d n, \gamma(f, n / m)} \geq d \varphi(n)$. The choice made for $y$ implies that the first term is negligible. For the second term observe that the Rankin Method (see [16, Lemma 3.3]) implies that for any $c \in(0,1)$, uniformly in $m$,

$$
\sum_{\substack{n \in \mathcal{S}_{m} \\ n \geq T}} \frac{1}{n} \ll_{c} \frac{1}{T^{c}}
$$

Hence

$$
\begin{aligned}
\tau(m) \frac{m}{\varphi(m)} \frac{x}{\log x} \sum_{\substack{n \in \mathcal{S}_{m}, n>y / m}} \frac{1}{\varphi(n)} & =\tau(m)\left(\frac{m}{\varphi(m)}\right)^{2} \frac{x}{\log x} \sum_{\substack{n \in \mathcal{S}_{m}, n>y / m}} \frac{1}{n} \\
& \leq \tau(m)\left(\frac{m}{\varphi(m)}\right)^{2} \frac{x}{\log x} \frac{m^{c}}{y^{c}} \\
& \ll \frac{\tau(m) m^{c} x(\log \log x)^{\frac{2 c}{3 r+3}}+2}{(\log x)^{1+\frac{c}{3 r+3}}} .
\end{aligned}
$$

Now let us deal with $\Sigma_{2}$. We have that

$$
\begin{aligned}
& \sum_{\substack{n \in \mathcal{S}_{m}, d \mid m \\
n m>y}} \sum_{\substack{d|n \\
f| n}} \pi_{\Gamma}\left(x, n d, \gamma\left(f, \frac{n}{m}\right)\right) \\
& \ll \tau(m)\left(\sum_{\substack{n \in \mathcal{S}_{m}, y<n m \leq z}} \sum_{d \mid m} \pi(x, n d, 1)+\sum_{\substack{n \in \mathcal{S}_{m}, n m>z}} \sum_{d \mid m} \#\{k \leq x: n d \mid k\}\right),
\end{aligned}
$$

where $z$ is a suitable parameter that will be determined momentarily. By the Brun-Tichmarch Theorem and the trivial estimate, the above is

$$
\ll \frac{\tau(m) m}{\varphi(m)} x\left(\frac{1}{\log (x / z)} \sum_{\substack{n \in \mathcal{S}_{m}, n m>y}} \frac{1}{\varphi(n)}+\sum_{\substack{n \in \mathcal{S}_{m}, n m>z}} \frac{1}{n}\right) .
$$

Applying one more (11), we obtain the estimate

$$
\Sigma_{2} \ll \tau(m)\left(\frac{m}{\varphi(m)}\right)^{2} m^{c} x\left(\frac{1}{\log (x / z) y^{c}}+\frac{1}{z^{c}}\right) .
$$

Finally, setting $z=\log ^{2+1 / c} x$ and $c=1-1 / \log \log x$ we obtain the claim. 
Proof of Theorem 2. We use the formulas for the degrees $k_{n d, \gamma\left(f, \frac{n}{m}\right)}(\Gamma)$ of Lemma 1 and of Corollary 1 which in this case reads as:

$$
k_{n d, \gamma\left(f, \frac{n}{m}\right)}(\Gamma)=\frac{d \varphi(n)}{\left|\mathcal{H}_{n d, v_{2}\left(\gamma\left(f, \frac{n}{m}\right)\right)}\right|} \prod_{\ell \mid f}\left|\Gamma\left(\ell^{v_{\ell}(n / m)+1}\right)\right|,
$$

where $\mathcal{H}_{n d, v_{2}\left(\gamma\left(f, \frac{n}{m}\right)\right)}$ is trivial if $f$ is odd while if $2 \mid f$, then $\left.v_{2}\left(\gamma\left(f, \frac{n}{m}\right)\right)=v_{2}\left(\frac{n}{m}\right)\right)+1$ and

$$
\mathcal{H}_{n d, v_{2}\left(\frac{n}{m}\right)+1}=\left\{\eta \in \mathbb{N}: \eta\left|\operatorname{Rad}(m), \eta^{2^{v_{2}\left(\frac{n}{m}\right)}} \mathbb{Q}^{* 2^{v_{2}\left(\frac{n}{m}\right)+1}} \in \Gamma\left(2^{v_{2}\left(\frac{n}{m}\right)+1}\right), \delta(\eta)\right| n d\right\} .
$$

Thus, if for brevity we write $v=v_{2}\left(\frac{n}{m}\right)$, the sum defining $\varrho_{\Gamma, m}$ in the statement of Theorem 1 equals

$$
\begin{aligned}
& \sum_{n \in \mathcal{S}_{m}} \frac{1}{\varphi(n)} \sum_{d \mid n} \frac{\mu(d)}{d} \sum_{f \mid n} \mu(f) \prod_{\ell \mid f}\left|\Gamma\left(\ell^{v_{\ell}(n / m)+1}\right)\right|^{-1} \\
& +\sum_{\substack{\eta \mid \operatorname{Rad}(m) \\
\eta \neq 1}} \sum_{\substack{n \in \mathcal{S}_{m} \\
\eta^{2} \mathbb{Q}^{* 2^{v+1}} \in \Gamma\left(2^{v+1}\right)}} \frac{1}{\varphi(n)} \sum_{\substack{d|n \\
\delta(\eta)| n d}} \frac{\mu(d)}{d} \sum_{\substack{f \mid n \\
f \text { even }}} \mu(f) \prod_{\ell \mid f}\left|\Gamma\left(\ell^{v_{\ell}(n / m)+1}\right)\right|^{-1} . \\
& =S_{1}+S_{2} \text {, }
\end{aligned}
$$

say. To compute $S_{1}$, we use the identity

$$
\frac{1}{\varphi(n)} \sum_{d \mid n} \frac{\mu(d)}{d}=\frac{1}{n}
$$

So that

$$
\begin{aligned}
S_{1} & =\sum_{n \in \mathcal{S}_{m}} \frac{1}{n} \prod_{\ell \mid m}\left(1-\left|\Gamma\left(\ell^{v_{\ell}(n / m)+1}\right)\right|^{-1}\right) \\
& =\prod_{\ell \mid m} \sum_{j \geq v_{\ell}(m)} \frac{1}{\ell^{j}}\left(1-\left|\Gamma\left(\ell^{j-v_{\ell}(m)+1}\right)\right|^{-1}\right) \\
& =\frac{1}{m} \prod_{\ell \mid m} \sum_{j \geq 0} \frac{1}{\ell^{j}}\left(1-\left|\Gamma\left(\ell^{j+1}\right)\right|^{-1}\right) \\
& =\frac{1}{\varphi(m)} \prod_{\ell \mid m}\left(1-(\ell-1) \sum_{j \geq 1} \frac{1}{\ell^{j}\left|\Gamma\left(\ell^{j}\right)\right|}\right) .
\end{aligned}
$$

We also deduce that for $m$ odd,

$$
\varrho_{\Gamma, m}=\frac{1}{\varphi(m)} \prod_{\ell \mid m}\left(1-\sum_{j \geq 1} \frac{\ell-1}{\ell^{j}\left|\Gamma\left(\ell^{j}\right)\right|}\right) .
$$

In order to compute $S_{2}$, we need to use the following lemma: 
Lemma 7. With the notation above, let

$$
S=\frac{1}{\varphi(n)} \sum_{\substack{d|n \\ \delta(\eta)| n d}} \frac{\mu(d)}{d}
$$

Then

$$
S=\frac{\tau_{\eta, n}}{n}, \quad \text { where } \quad \tau_{\eta, n}= \begin{cases}1 & \text { if } \delta(\eta) \mid n, \\ -1 & \text { if } \delta(\eta) \nmid n \text { but } \delta(\eta) \mid 2 n, \\ 0 & \text { if } \delta(\eta) \nmid 2 n .\end{cases}
$$

Proof of Lemma 7. Set $\delta(\eta)=x 2^{\beta}$ with $x$ odd squarefree and $\beta \in\{0,2,3\}$. Furthermore, set $n=n^{\prime} 2^{\alpha}$ with $n^{\prime}$ odd.

The condition $\delta(\eta) \mid n$ implies that $\delta(\eta) \mid n d$ for all possible $d$ and in such a case, we have that $S=\frac{1}{n}$ by the multiplicativity of the involved functions.

The condition $\delta(\eta) \nmid n, \delta(\eta) \mid 2 n$ is equivalent to $x \mid n^{\prime}$ and $\beta=\alpha+1$, which in particular, implies that $n$ is even. Therefore, in this case, by multiplicativity,

$$
S=\frac{1}{n^{\prime}} \times \frac{1}{2^{\alpha-1}} \sum_{\substack{\gamma \in\{0,1\}, \beta \leq \alpha+\gamma}} \frac{(-1)^{\gamma}}{2^{\gamma}}=-\frac{1}{n} .
$$

Finally, if the condition $\delta(\eta) \nmid 2 n$ is satisfied, since $x \nmid n^{\prime}$, for all squarefree $d \mid n$, we have that $\delta(\eta) \nmid n d$ so, in such a case, $S=0$. So we can assume that $x \mid n^{\prime}$, $\beta>\alpha+1$ and that $\beta \in\{2,3\}$. It follows that

$$
S=\frac{1}{n^{\prime}} \times \frac{1}{\varphi\left(2^{\alpha}\right)} \sum_{\substack{\gamma \in\{0,1\}, \alpha+1<\beta \leq \alpha+\gamma}} \frac{(-1)^{\gamma}}{2^{\gamma}}=0,
$$

since the conditions on $\gamma$ in the sum are never satisfied. This concludes the proof.

Next, note that $S_{2}=0$ unless $m$ is even. In the latter case we write

$$
S_{2}=\sum_{\substack{\eta \mid \operatorname{Rad}(m) \\ \eta \neq 1}} S_{\eta}
$$

where, by Lemma 7

$$
S_{\eta}=\sum_{\substack{n \in \mathcal{S}_{m} \\ \eta^{2^{v_{2}(n / m)}}}} \frac{\tau_{\eta, n}}{n} \sum_{\substack{f \mid n \\ \mathbb{Q}^{v_{2}\left(\frac{n}{m}\right)+1} \in \Gamma\left(2^{v_{2}(n / m)+1}\right)}} \mu(f) \prod_{\ell \mid f}\left|\Gamma\left(\ell^{v_{\ell}(n / m)+1}\right)\right|^{-1} .
$$


Next we use the fact that $S_{\eta}=0$ unless $\delta(\eta) \mid 2 n$ and this happens only if $\eta \mid m$. Furthermore, $S_{\eta}=0$ unless there exists $t \geq 0$ such that $\eta^{2^{t}} \mathbb{Q}^{* 2^{t+1}} \in \Gamma\left(2^{t+1}\right)$. We will set $t_{\eta}$ to be the least of such $t$ so that $t_{\eta}=\infty$ if there is no $t$ with such a property. Furthermore, if $s \geq t_{\eta}$, then $\eta^{2^{s}} \mathbb{Q}^{* 2^{s+1}} \in \Gamma\left(2^{s+1}\right)$.

Hence, for $m$ even, we can rewrite

$$
S_{2}=\sum_{\substack{\eta \mid \operatorname{Rad}(m), \eta \neq 1 \\ t_{\eta}<\infty}} S_{\eta}
$$

We deduce that if $S_{\eta}$ is one of the summands above, then it equals

$$
\begin{aligned}
- & \sum_{\substack{n \in \mathcal{S}_{m} \\
v_{2}(n / m) \geq t_{\eta}}} \frac{\tau_{\eta, n}}{n\left|\Gamma\left(2^{v_{2}(n / m)+1}\right)\right|} \prod_{\substack{\ell \mid n \\
\ell>2}}\left(1-\left|\Gamma\left(\ell^{v_{\ell}(n / m)+1}\right)\right|^{-1}\right) \\
= & \sum_{\substack{n \in \mathcal{S}_{m} \\
v_{2}(\delta(\eta)) \leq v_{2}(n)+1 \\
v_{2}(n / m) \geq t_{\eta}}} \frac{\epsilon_{\eta}\left(v_{2}(n)\right)}{n\left|\Gamma\left(2^{v_{2}(n / m)+1}\right)\right|} \prod_{\substack{\ell \mid n \\
\ell>2}}\left(1-\left|\Gamma\left(\ell^{v_{\ell}(n / m)+1}\right)\right|^{-1}\right),
\end{aligned}
$$

where $\epsilon_{\eta}(j)=1$ if $j=v_{2}(\delta(\eta) / 2)$ and $\epsilon_{\eta}(j)=-1$ if $j>v_{2}(\delta(\eta) / 2)$. So $S_{\eta}$ equals

$$
\begin{aligned}
& S_{1} \times 2^{v_{2}(m)-1}\left(1-\sum_{j \geq 1} \frac{1}{2^{j}\left|\Gamma\left(2^{j}\right)\right|}\right)^{-1} \times \sum_{\substack{j \geq t_{\eta}+v_{2}(m) \\
j \geq v_{2}\left(\frac{\delta(\eta)}{2}\right)}} \frac{\epsilon_{\eta}(j)}{2^{j}\left|\Gamma\left(2^{j-v_{2}(m)+1}\right)\right|} \\
= & S_{1} \times\left(1-\sum_{j \geq 1} \frac{1}{2^{j}\left|\Gamma\left(2^{j}\right)\right|}\right)^{-1} \times \sum_{k \geq \max \left\{t_{\eta}+1, v_{2}(\delta(\eta) / m)\right\}} \frac{\epsilon_{\eta}\left(k+v_{2}(m / 2)\right)}{2^{k}\left|\Gamma\left(2^{k}\right)\right|} .
\end{aligned}
$$

Hence,

$$
\varrho_{\Gamma, m}=\frac{1}{\varphi(m)} \prod_{\ell \mid m}\left(1-\sum_{j \geq 1} \frac{\ell-1}{\ell^{j}\left|\Gamma\left(\ell^{j}\right)\right|}\right) \times \nu_{\Gamma, m},
$$

where, if $m$ is odd, $\nu_{\Gamma, m}=1$ and, if $m$ is even, $\nu_{\Gamma, m}$ equals

$$
1+\left(1-\sum_{j \geq 1} \frac{1}{2^{j}\left|\Gamma\left(2^{j}\right)\right|}\right)^{-1} \sum_{\substack{\eta \mid \operatorname{Rad}(m) \\ \eta \neq 1 \\ t_{\eta}<\infty}} \sum_{\substack{k \geq t_{\eta}+1 \\ k \geq v_{2}(\delta(\eta) / m)}} \frac{\epsilon_{\eta}\left(k+v_{2}(m / 2)\right)}{2^{k}\left|\Gamma\left(2^{k}\right)\right|} .
$$


If we add to the last sum above the term $\eta=1$ and we observe that

$$
-\sum_{\substack{k \geq t_{1}+1 \\ k \geq v_{2}(\delta(1) / m)}} \frac{\epsilon_{1}\left(k+v_{2}(m / 2)\right)}{2^{k}\left|\Gamma\left(2^{k}\right)\right|}=\sum_{j \geq 1} \frac{1}{2^{j}\left|\Gamma\left(2^{j}\right)\right|}
$$

since $t_{1}=0, \delta(1)=1$ and $\epsilon_{1}\left(k+v_{2}(m / 2)\right)=-1$, we mildly simplify the formula for $\nu_{\Gamma, m}$ when $m$ is even, obtaining:

$$
\begin{aligned}
\nu_{\Gamma, m} & =\left(1-\sum_{j \geq 1} \frac{1}{2^{j}\left|\Gamma\left(2^{j}\right)\right|}\right)^{-1}\left(1+\sum_{\substack{\eta \mid \operatorname{Rad}(m) \\
t_{\eta}<\infty}} \sum_{\substack{k \geq t_{\eta}+1 \\
k \geq s_{\eta}}} \frac{\epsilon_{\eta}\left(k+v_{2}\left(\frac{m}{2}\right)\right)}{2^{k}\left|\Gamma\left(2^{k}\right)\right|}\right) . \\
& =\left(1-\sum_{j \geq 1} \frac{1}{2^{j}\left|\Gamma\left(2^{j}\right)\right|}\right)^{-1}\left(1-\sum_{\eta \mid \operatorname{Rad}(m)} \psi_{\eta}\right),
\end{aligned}
$$

where $s_{\eta}=v_{2}\left(\frac{\delta(\eta)}{m}\right)$ and

$$
\psi_{\eta}= \begin{cases}0 & \text { if } t_{\eta}=\infty, \\ \sum_{k>t_{\eta}} \frac{1}{2^{k}\left|\Gamma\left(2^{k}\right)\right|} & \text { if } s_{\eta} \leq t_{\eta}<\infty, \\ -\frac{1}{2^{s_{\eta}}\left|\Gamma\left(2^{s_{\eta}}\right)\right|}+\sum_{k>s_{\eta}} \frac{1}{2^{k}\left|\Gamma\left(2^{k}\right)\right|} & \text { if } s_{\eta}>t_{\eta},\end{cases}
$$

and this completes the proof.

\section{NUMERICAL DATA}

In this section we compare numerical data. The density $\varrho_{\Gamma, m}$ can be explicitly computed once a set of generators of $\Gamma$ is given. In particular, the following Pari-GP 21] code allows us to compute $\varrho_{\left\langle p_{1}, \cdots, p_{r}\right\rangle, m}=$ rho(m,p_1 $\cdots$ p_r).

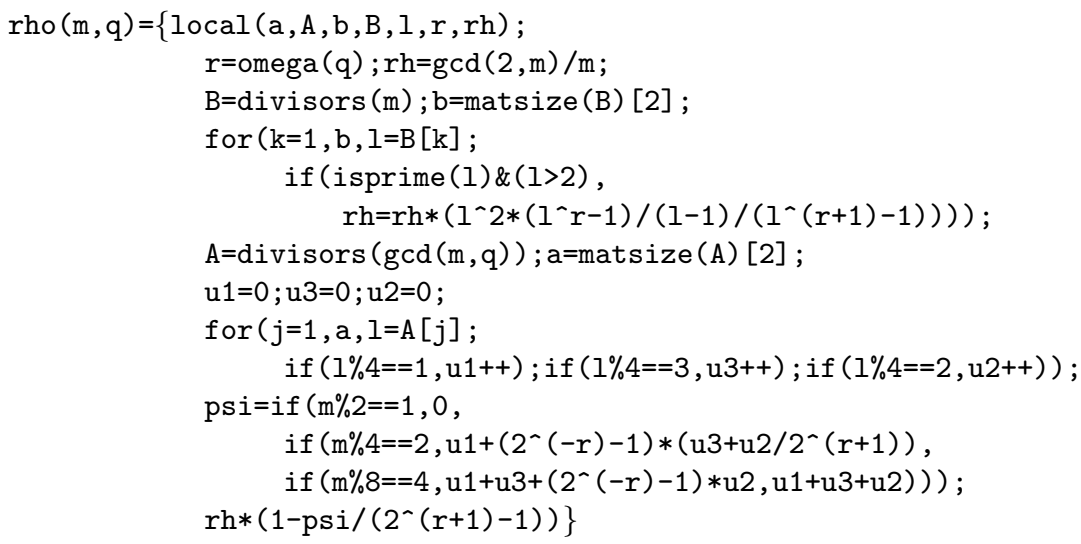


The first table compares the values of $\varrho_{\Gamma_{r}, m}$ as in Theorem 1 (second row) and $\frac{A_{\Gamma_{r}}\left(10^{9}, m\right)}{\pi\left(10^{9}\right)}$ (first row) with $\Gamma_{r}=\left\langle 2, \ldots, p_{r}\right\rangle, r \leq 7\left(p_{i}\right.$ is the $i$-th prime) and $m=2, \ldots, 16$. All values have been truncated to 7 decimal digits.

\begin{tabular}{|c|c|c|c|c|c|c|c|}
\hline$m \backslash \Gamma_{r}$ & 1 & 2 & 3 & 4 & 5 & 6 & 7 \\
\hline \multirow[t]{2}{*}{2} & 0.7083259 & 0.8705329 & 0.9369869 & 0.9686946 & 0.9843725 & 0.9921912 & 0.9960977 \\
\hline & 0.7083333 & 0.8705357 & 0.9369791 & 0.9686869 & 0.9843672 & 0.9921865 & 0.9960936 \\
\hline \multirow[t]{2}{*}{3} & 0.3750162 & 0.4615489 & 0.4874978 & 0.4958546 & 0.4986178 & 0.4995315 & 0.4998315 \\
\hline & 0.3750000 & 0.4615384 & 0.4875000 & 0.4958677 & 0.4986263 & 0.4995425 & 0.4998475 \\
\hline \multirow[t]{2}{*}{4} & 0.4166745 & 0.4821469 & 0.4958488 & 0.4989975 & 0.4997547 & 0.4999387 & 0.4999818 \\
\hline & 0.4166666 & 0.4821428 & 0.4958333 & 0.4989919 & 0.4997519 & 0.4999384 & 0.4999846 \\
\hline \multirow[t]{2}{*}{5} & 0.2083311 & 0.2419332 & 0.2483914 & 0.2496736 & 0.2499273 & 0.2499772 & 0.2499875 \\
\hline & 0.2083333 & 0.2419354 & 0.2483974 & 0.2496798 & 0.2499359 & 0.2499871 & 0.2499974 \\
\hline \multirow[t]{2}{*}{6} & 0.2656511 & 0.4574280 & 0.4869920 & 0.4957940 & 0.4986109 & 0.4995309 & 0.4998313 \\
\hline & 0.2656250 & 0.4574175 & 0.4869921 & 0.4958052 & 0.4986186 & 0.4995415 & 0.4998474 \\
\hline \multirow[t]{2}{*}{7} & 0.1458489 & 0.1637375 & 0.1662449 & 0.1665994 & 0.1666516 & 0.1666582 & 0.1666592 \\
\hline & 0.1458333 & 0.1637426 & 0.1662500 & 0.1666071 & 0.1666581 & 0.1666654 & 0.1666664 \\
\hline \multirow[t]{2}{*}{8} & 0.0833265 & 785587 & 166697 & 8669 & 20661 & 2460616 & 0.2480390 \\
\hline & 0.0833333 & 0.1785714 & 0.2166666 & 0.2338709 & 0.2420634 & 0.2460629 & 0.2480392 \\
\hline \multirow[t]{2}{*}{9} & 0.1249966 & 0.1538451 & 0.1625054 & 0.1652942 & 0.1662133 & 0.1665179 & 0.1666177 \\
\hline & 0.1250000 & 0.1538461 & 0.1625000 & 0.1652892 & 0.1662087 & 0.1665141 & 0.1666158 \\
\hline \multirow[t]{2}{*}{10} & 0.1475587 & 0.2106102 & 0.2170853 & 0.2340359 & 0.2421145 & 0.2460758 & 0.2480397 \\
\hline & 0.1475694 & 0.2106134 & 0.2170890 & 0.2340434 & 0.2421216 & 0.2460806 & 0.2480442 \\
\hline \multirow[t]{2}{*}{11} & 0.0916644 & 0.0992460 & 0.0999258 & 0.0999871 & 0.0999930 & 0.0999937 & 0.0999937 \\
\hline & 0.0916666 & 0.0992481 & 0.0999316 & 0.0999937 & 0.0999994 & 0.0999999 & 0.0999999 \\
\hline \multirow[t]{2}{*}{12} & & & 0.2396969 & & & & 0.2498959 \\
\hline & 0.1562500 & 0.2142857 & 0.2396875 & 0.2469341 & 0.2490658 & 0.2497098 & 0.2499084 \\
\hline \multirow[t]{2}{*}{13} & 0.0773848 & 0.0828743 & 0.0832971 & 0.0833291 & 0.0833317 & 0.0833320 & 0.0833320 \\
\hline & 0.0773809 & 0.0828779 & 0.0832983 & 0.0833306 & 0.0833331 & 0.0833333 & 0.0833333 \\
\hline \multirow[t]{2}{*}{14} & 0.1033220 & 0.1425403 & 0.1557674 & 0.1665792 & 0.1666493 & 0.1666580 & 0.1666592 \\
\hline & 0.1032986 & 0.1425438 & 0.1557727 & 0.1665861 & 0.1666555 & 0.1666651 & 0.1666664 \\
\hline \multirow[t]{2}{*}{15} & 0.0781280 & 0.1116612 & 0.1210907 & 0.1238016 & 0.1246141 & 0.1248689 & 0.1249475 \\
\hline & 0.0781250 & 0.1116625 & 0.1210937 & 0.1238082 & 0.1246246 & 0.1248792 & 0.1249606 \\
\hline \multirow[t]{2}{*}{16} & 0.0416661 & 0.0892749 & 0.1083288 & 0.1169345 & 0.1210315 & 0.1230292 & 0.1240151 \\
\hline & 0.0416666 & 0.0892857 & 0.1083333 & 0.1169354 & 0.1210317 & 0.1230314 & 0.1240196 \\
\hline
\end{tabular}


The next table compares the values of $\varrho_{\tilde{\Gamma}_{r}, m}$ as in Theorem 1 (second row) and $\frac{A_{\tilde{\Gamma}_{r}}\left(10^{9}, m\right)}{\pi\left(10^{9}\right)}$ (first row) with $\tilde{\Gamma}_{r}=\left\langle 3, \ldots, p_{r+1}\right\rangle, r \leq 7$ and $2 \leq m \leq 16$.

\begin{tabular}{|c|c|c|c|c|c|c|c|}
\hline$m \backslash \tilde{\Gamma}_{r}$ & 1 & 2 & 3 & 4 & 5 & 6 & 7 \\
\hline \multirow[t]{2}{*}{2} & 0.6666655 & 0.8571448 & 0.9333310 & 0.9677335 & 0.9841212 & 0.9921209 & 0.9960788 \\
\hline & 0.6666666 & 0.8571428 & 0.9333333 & 0.9677419 & 0.9841269 & 0.9921259 & 0.9960784 \\
\hline \multirow[t]{2}{*}{3} & 0.3749919 & 0.4615306 & 0.4874732 & 0.4958573 & 0.4986160 & 0.4995291 & 0.4998312 \\
\hline & 0.3750000 & 0.4615384 & 0.4875000 & 0.4958677 & 0.4986263 & 0.4995425 & 0.4998475 \\
\hline \multirow[t]{2}{*}{4} & 0.3333555 & 0.4285866 & 0.4666680 & 0.4838841 & 0.4920754 & 0.4960635 & 0.4980383 \\
\hline & 0.3333333 & 0.4285714 & 0.4666666 & 0.4838709 & 0.4920634 & 0.4960629 & 0.4980392 \\
\hline \multirow[t]{2}{*}{5} & 0.2083280 & 0.2419252 & 0.2484011 & 0.2496762 & 0.2499270 & 0.2499777 & 0.2499876 \\
\hline & 0.2083333 & 0.2419354 & 0.2483974 & 0.2496798 & 0.2499359 & 0.2499871 & 0.2499974 \\
\hline \multirow[t]{2}{*}{6} & 0.3124943 & 0.4450448 & 0.4834115 & 0.4948565 & 0.4983659 & 0.4994672 & 0.4998148 \\
\hline & 0.3125000 & 0.4450549 & 0.4834375 & 0.4948680 & 0.4983790 & 0.4994810 & 0.4998322 \\
\hline \multirow[t]{2}{*}{7} & 0.1458220 & 0.1637352 & 0.1662398 & 0.1666008 & 0.1666509 & 0.1666581 & 0.1666592 \\
\hline & 0.1458333 & 0.1637426 & 0.1662500 & 0.1666071 & 0.1666581 & 0.1666654 & 0.1666664 \\
\hline \multirow[t]{2}{*}{8} & 0.1666562 & 0.2142934 & 0.2333303 & 0.2419403 & 0.2460312 & 0.2480318 & 0.2490220 \\
\hline & 0.1666666 & 0.2142857 & 0.2333333 & 0.2419354 & 0.2460317 & 0.2480314 & 0.2490196 \\
\hline \multirow[t]{2}{*}{9} & 0.1250027 & 0.1538590 & 0.1625073 & 0.1652946 & 0.1662161 & 0.1665172 & 0.1666171 \\
\hline & 0.1250000 & 0.1538461 & 0.1625000 & 0.1652892 & 0.1662087 & 0.1665141 & 0.1666158 \\
\hline \multirow[t]{2}{*}{10} & 0.1388773 & 0.1728045 & 0.2152763 & 0.2335623 & 0.2419895 & 0.2460393 & 0.2480265 \\
\hline & 0.1388888 & 0.1728110 & 0.2152777 & 0.2335715 & 0.2420015 & 0.2460503 & 0.2480366 \\
\hline \multirow[t]{2}{*}{11} & 0.0916609 & 0.0992403 & 0.0999244 & 0.0999869 & 0.0999931 & 0.0999936 & 0.0999937 \\
\hline & 0.0916666 & 0.0992481 & 0.0999316 & 0.0999937 & 0.0999994 & 0.0999999 & 0.0999999 \\
\hline \multirow[t]{2}{*}{12} & 0.0624985 & 0.1648314 & 0.2112409 & 0.2319473 & 0.2414047 & 0.2458287 & 0.2479503 \\
\hline & 0.0625000 & 0.1648351 & 0.2112500 & 0.2319381 & 0.2413984 & 0.2458378 & 0.2479635 \\
\hline \multirow[t]{2}{*}{13} & 0.0773695 & 0.0828785 & 0.0832960 & 0.0833287 & 0.0833318 & 0.0833320 & 0.0833320 \\
\hline & 0.0773809 & 0.0828779 & 0.0832983 & 0.0833306 & 0.0833331 & 0.0833333 & 0.0833333 \\
\hline \multirow[t]{2}{*}{14} & 0.0972166 & 0.1403456 & 0.1648538 & 0.1662621 & 0.1665672 & 0.1666369 & 0.1666534 \\
\hline & 0.0972222 & 0.1403508 & 0.1648645 & 0.1662712 & 0.1665754 & 0.1666449 & 0.1666613 \\
\hline \multirow[t]{2}{*}{15} & 0.0781188 & 0.1116473 & 0.1210896 & 0.1238047 & 0.1246196 & 0.1248686 & 0.1249482 \\
\hline & 0.0781250 & 0.1116625 & 0.1210937 & 0.1238082 & 0.1246246 & 0.1248792 & 0.1249606 \\
\hline \multirow[t]{2}{*}{16} & 0.0833204 & 0.1071366 & 0.1166656 & 0.1209677 & 0.1230143 & 0.1240113 & 0.1245069 \\
\hline & 0.0833333 & 0.1071428 & 0.1166666 & 0.1209677 & 0.1230158 & 0.1240157 & 0.1245098 \\
\hline
\end{tabular}


The next table compares the values of $\beta_{\Gamma_{r}, k}$ (i.e., the density of primes $p$ with $\operatorname{ord}_{p}\left(\Gamma_{r}\right) k$-free) (first row) and $\frac{\#\left\{p \leq 10^{9}, p \notin \operatorname{Supp}^{\prime}, \operatorname{ord}_{p}(\Gamma) \text { is } k \text {-free }\right\}}{\pi\left(10^{9}\right)}$ (second row) for $k=2, \ldots, 7$ and $r=1, \ldots, 7$.

\begin{tabular}{|c|c|c|c|c|c|c|c|}
\hline$k \backslash \Gamma_{r}$ & 1 & 2 & 3 & 4 & 5 & 6 & 7 \\
\hline & 0.4643728 & 0.3916870 & 0.3783724 & 0.3751626 & 0.3743029 & 0.3740588 & 0.3739871 \\
2 & 0.4643773 & 0.3916738 & 0.3783458 & 0.3751487 & 0.3742881 & 0.3740453 & 0.3739753 \\
\hline & 0.8669787 & 0.7640822 & 0.7275550 & 0.7117925 & 0.7044658 & 0.7009347 & 0.6992045 \\
3 & 0.8669801 & 0.7640826 & 0.7275397 & 0.7117918 & 0.7044620 & 0.7009346 & 0.6992023 \\
\hline & 0.9429226 & 0.8922523 & 0.8729475 & 0.8644050 & 0.8603871 & 0.8584410 & 0.8574845 \\
4 & 0.9429270 & 0.8922653 & 0.8729480 & 0.8644003 & 0.8603827 & 0.8584393 & 0.8574853 \\
\hline & 0.9742393 & 0.9493687 & 0.9396381 & 0.9352925 & 0.9332389 & 0.9322416 & 0.9317506 \\
5 & 0.9742428 & 0.9493723 & 0.9396454 & 0.9352960 & 0.9332398 & 0.9322460 & 0.9317542 \\
\hline & 0.9879809 & 0.9757187 & 0.9708684 & 0.9686929 & 0.9676621 & 0.9671607 & 0.9669135 \\
6 & 0.9879833 & 0.9757210 & 0.9708738 & 0.9687015 & 0.9676725 & 0.9671724 & 0.9669251 \\
\hline & 0.9942653 & 0.9881936 & 0.9857800 & 0.9846948 & 0.9841798 & 0.9839289 & 0.9838052 \\
7 & 0.9942667 & 0.9881987 & 0.9857830 & 0.9846992 & 0.9841872 & 0.9839368 & 0.9838137 \\
\hline & 0.9972219 & 0.9942060 & 0.9930041 & 0.9924629 & 0.9922058 & 0.9920804 & 0.9920185 \\
8 & 0.9972247 & 0.9942058 & 0.9930081 & 0.9924704 & 0.9922122 & 0.9920868 & 0.9920254 \\
\hline
\end{tabular}

Example. Let $\Gamma=\left\langle 3^{3} \cdot 11^{15}, 3^{3} \cdot 11^{3}, 3^{7} \cdot 13^{7}, 2^{2} \cdot 5^{2} \cdot 11 \cdot 13\right\rangle$. Then $\operatorname{Supp}(\Gamma)=$ $(2,3,5,11,13)$ and the matrix associated to $\Gamma$ is

$$
M=\left(\begin{array}{cccc}
0 & 0 & 0 & 2 \\
3 & 3 & 7 & 0 \\
0 & 0 & 0 & 2 \\
15 & 3 & 0 & 1 \\
0 & 0 & 7 & 1
\end{array}\right)
$$

so $\Delta_{4}(\Gamma)=2^{3} \cdot 3^{2} \cdot 7, \Delta_{3}(\Gamma)=2 \cdot 3$ and $\Delta_{2}(\Gamma)=\Delta_{1}(\Gamma)=1$. Hence, if $\ell \nmid 42$, then

$$
1-\sum_{j \geq 1} \frac{\ell-1}{\ell^{j}\left|\Gamma\left(\ell^{j}\right)\right|}=\frac{\ell\left(\ell^{4}-1\right)}{\ell^{5}-1}
$$

while

$$
1-\sum_{j \geq 1} \frac{2}{3^{j}\left|\Gamma\left(3^{j}\right)\right|}=\frac{2^{4} \times 21}{3 \times 11^{2}} \quad \text { and } \quad 1-\sum_{j \geq 1} \frac{6}{7^{j}\left|\Gamma\left(7^{j}\right)\right|}=\frac{2 \times 11 \times 127}{2801} .
$$

Furthermore, if $\eta$ is squarefree and $t_{\eta}$ is finite (i.e., $\eta^{2^{t}} \mathbb{Q}^{* 2^{t+1}} \in \Gamma\left(2^{t+1}\right)$ for some $t \geq 0$ ), then $\eta \mid 2 \times 3 \times 5 \times 11 \times 13$. More precisely, after some calculations, one obtains that

$$
t_{\eta}= \begin{cases}0 & \text { if } \eta \in\{1,33,39,143\} \\ 1 & \text { if } \eta \in\{30,110,130,4290\} \\ 2 & \text { if } \eta \in\{3,11,10,13,330,390,1430\} \\ \infty & \text { otherwise }\end{cases}
$$

So by (5)

$$
\sum_{j \geq j_{0}} \frac{1}{2^{j}\left|\Gamma\left(2^{j}\right)\right|}= \begin{cases}\frac{33}{2^{3} \times 31} & \text { if } j_{0}=1 \\ \frac{1}{2^{2} \times 31} & \text { if } j_{0}=2 \\ \frac{1}{2^{7} \times 31} & \text { if } j_{0}=3\end{cases}
$$


We conclude that

$$
\psi_{\eta}= \begin{cases}\frac{33}{2^{3} \times 31} & \text { if } \eta \in\{1,33\} \text { or if } \eta \in\{39,143\} \text { and } 4 \mid m, \\ \frac{1}{2^{2} \times 31} & \text { if } \eta \in\{30,110,130,4290\} \text { and } 4 \mid m, \\ \frac{1}{2^{7} \times 31} & \text { if } \eta \in\{3,11,10,13,330,390,1430\} \\ -\frac{29}{2^{3} \times 31} & \text { if } \eta \in\{39,143\} \text { and } 2 \| m, \\ -\frac{15}{2^{6} \times 31} & \text { if } \eta \in\{30,110,130,4290\} \text { and } 2 \| m, \\ 0 & \text { otherwise. }\end{cases}
$$

The following table compares the values of $\varrho_{\Gamma, m}$ as in Theorem 1 (second row) and $\frac{A_{\Gamma}\left(10^{9}, m\right)}{\pi\left(10^{9}\right)}$ (first row) with $\Gamma$ and $m=2, \ldots, 25$. The numbers are truncated (not approximated) to the seventh decimal digit.

\begin{tabular}{|c|c|c|c|c|c|c|c|}
\hline$m$ & 2 & 3 & 4 & 5 & 6 & 7 & 8 \\
\hline & 0.86691300 & 0.46280353 & 0.43348907 & 0.24967274 & 0.40110378 & 0.16624556 & 0.21673147 \\
& 0.86693548 & 0.46280992 & 0.43346774 & 0.24967990 & 0.40110970 & 0.16625015 & 0.21673387 \\
\hline$m$ & 9 & 10 & 11 & 12 & 13 & 14 & 15 \\
\hline & 0.15427696 & 0.21638900 & 0.09998758 & 0.20057942 & 0.08332899 & 0.14412518 & 0.11554303 \\
& 0.15426997 & 0.21639344 & 0.09999379 & 0.20055485 & 0.08333064 & 0.14412815 & 0.11555433 \\
\hline$m$ & 16 & 17 & 18 & 19 & 20 & 21 & 22 \\
\hline & 0.10836781 & 0.06248592 & 0.13371134 & 0.05554725 & 0.10819549 & 0.07695901 & 0.08666158 \\
& 0.10836694 & 0.06249929 & 0.13374211 & 0.05555515 & 0.10822818 & 0.07694221 & 0.08666296 \\
\hline$m$ & 23 & 24 & 25 & 26 & 27 & 28 & 29 \\
\hline & 0.04544655 & 0.10028492 & 0.04993461 & 0.07222781 & 0.05141541 & 0.07206581 & 0.03571052 \\
& 0.04545439 & 0.10027743 & 0.04993598 & 0.07222128 & 0.05142332 & 0.07206407 & 0.03571423 \\
\hline$m$ & 30 & 31 & 32 & 33 & 34 & 35 & 36 \\
\hline & 0.10098433 & 0.03332901 & 0.05418229 & 0.04627953 & 0.05417804 & 0.04149951 & 0.066869103 \\
& 0.10099355 & 0.03333329 & 0.05418346 & 0.04627811 & 0.05418285 & 0.04150932 & 0.066871057 \\
\hline \hline$m$ & 37 & 38 & 39 & 40 & 41 & 42 & 43 \\
\hline & 0.02777853 & 0.04815382 & 0.03856533 & 0.05408612 & 0.02500475 & 0.06670581 & 0.023815314 \\
& 0.02777776 & 0.04816273 & 0.03856624 & 0.05409836 & 0.02499999 & 0.06668454 & 0.023809517 \\
\hline
\end{tabular}

\section{Conclusion}

Average values of $\operatorname{ord}_{p}(\Gamma)$ in the sense of Kurlberg and Pomerance [8] or weighted sum of $\operatorname{ind}_{p}(\Gamma)$ in the sense of [14] can also be considered. For example, if $m \in \mathbb{N}$, in [17. Susa and the author consider the problem of enumerating primes $p$ such that $\operatorname{ind}_{p}(\Gamma)=m$.

\section{ACKNOWLEDGEMENTS}

The author would like to thank Pieter Moree and one anonymous referee for several helpful remarks and suggestions. 


\section{REFERENCES}

[1] Leonardo Cangelmi and Francesco Pappalardi, On the $r$-rank Artin conjecture. II, J. Number Theory 75 (1999), no. 1, 120-132, DOI 10.1006/jnth.1998.2319. MR1677559 (2000i:11149).

[2] Koji Chinen and Leo Murata, On a distribution property of the residual order of a $(\bmod p)$. $I V$, Number theory, Dev. Math., vol. 15, Springer, New York, 2006, pp. 11-22, DOI 10.1007/0387-30829-6_2. MR2213825 (2008a:11120)

[3] Rajiv Gupta and M. Ram Murty, A remark on Artin's conjecture, Invent. Math. 78 (1984), no. 1, 127-130, DOI 10.1007/BF01388719. MR762358 (86d:11003)

[4] Rajiv Gupta and M. Ram Murty, Primitive points on elliptic curves, Compositio Math. 58 (1986), no. 1, 13-44. MR834046 (87h:11050)

[5] Helmut Hasse, Über die Dichte der Primzahlen p, für die eine vorgegebene ganzrationale Zahl $a \neq 0$ von gerader bzw.ungerader Ordnung mod. $p$ ist (German), Math. Ann. 166 (1966), 19-23. MR0205975 (34 \#5800)

[6] D. R. Heath-Brown, Artin's conjecture for primitive roots, Quart. J. Math. Oxford Ser. (2) 37 (1986), no. 145, 27-38, DOI 10.1093/qmath/37.1.27. MR830627 (88a:11004)

[7] Christopher Hooley, On binary cubic forms, J. Reine Angew. Math. 226 (1967), 30-87. MR0213299 (35 \#4163)

[8] P. Kurlberg and C. Pomerance, On a problem of Arnold: the average multiplicative order of a given integer, Algebra and Number Theory, 7 (2013), no. 4, 981-999. MR3095233

[9] J. C. Lagarias and A. M. Odlyzko, Effective versions of the Chebotarev density theorem, Algebraic number fields: $L$-functions and Galois properties (Proc. Sympos., Univ. Durham, Durham, 1975), Academic Press, London, 1977, pp. 409-464. MR0447191 (56 \#5506)

[10] Serge Lang, Algebra, Addison-Wesley Publishing Co., Inc., Reading, Mass., 1965. MR.0197234 $(33$ \#5416)

[11] Pieter Moree, Artin's primitive root conjecture-a survey, Integers 12 (2012), no. 6, 13051416, DOI 10.1515/integers-2012-0043. MR3011564

[12] Pieter Moree, On primes $p$ for which d divides $\operatorname{ord}_{p}(g)$, Funct. Approx. Comment. Math. 33 (2005), 85-95. MR2274151 (2007j:11131)

[13] Pieter Moree, On the distribution of the order over residue classes, Electron. Res. Announc. Amer. Math. Soc. 12 (2006), 121-128 (electronic), DOI 10.1090/S1079-6762-06-001685. MR2263073 (2007e:11117)

[14] F. Pappalardi, On Hooley's theorem with weights, Rend. Sem. Mat. Univ. Politec. Torino 53 (1995), no. 4, 375-388. Number theory, II (Rome, 1995). MR1452393 (98c:11102)

[15] Francesco Pappalardi, On the r-rank Artin conjecture, Math. Comp. 66 (1997), no. 218, 853-868, DOI 10.1090/S0025-5718-97-00805-3. MR1377664 (97f:11082)

[16] Francesco Pappalardi, Square free values of the order function, New York J. Math. 9 (2003), 331-344. MR2028173 (2004i:11116)

[17] F. Pappalardi and A. Susa, An analogue of Artin's Conjecture for multiplicative subgroups, Arch. Math. 101 (2013), no. 4, 319-330. MR3116653

[18] Jean-Pierre Serre, Quelques applications du théorème de densité de Chebotarev (French), Inst. Hautes Études Sci. Publ. Math. 54 (1981), 323-401. MR644559 (83k:12011)

[19] Edwin Weiss, Algebraic Number Theory, McGraw-Hill Book Co., Inc., New York, 1963. MR.0159805 (28 \#3021)

[20] K. Wiertelak, On the density of some sets of primes. IV, Acta Arith. 43 (1984), no. 2, 177-190. MR736730 (86e:11081)

[21] PARI/GP, version 2.3.4, http://pari.math.u-bordeaux.fr/, Bordeaux, 2009.

Dipartimento di Matematica e Fisica, Università Roma Tre, Largo S. L. Murialdo 1, I-00146, Roma, ItAly

E-mail address: pappa@mat.uniroma3.it 